\title{
Advanced numerical method for a thermally induced slug flow: application to a capillary Closed Loop Pulsating Heat Pipe
}

\author{
M. Manzoni ${ }^{1, *}$, M. Mameli ${ }^{2}$, C. de Falco ${ }^{3}$, L. Araneo ${ }^{4}$, S. Filippeschi ${ }^{2}$, and M. Marengo ${ }^{1,5}$ \\ ${ }^{1}$ Università degli Studi di Bergamo, Department of Engineering and Applied Sciences, Viale \\ Marconi 5, 24044 Dalmine (BG), Italy \\ ${ }^{2}$ Università di Pisa, DESTEC, Largo Lazzarino 2, 56122 Pisa, Italy \\ ${ }^{3}$ Politecnico di Milano, MOX, Modeling and Scientific Computing, Department of Mathematics, \\ Piazza Leonardo da Vinci 32, 20133 Milano, Italy \\ ${ }^{4}$ Politecnico di Milano, Energy Department, Via Lambruschini 4A, 20158 Milano, Italy \\ ${ }^{5}$ University of Brighton, School of Computing, Engineering and Mathematics, Lewes Road, BN2 \\ 4GJ, Brighton, $U K$
}

\begin{abstract}
An advanced hybrid lumped parameter code for the simulation of Pulsating Heat Pipes is developed. Being able to simulate transient operative conditions and removing common physical simplified assumptions, it represents a step forward with respect to the present models of passive two-phase systems. Mass, momentum and energy balances account for the thermal and fluiddynamics phenomena. Heterogeneous and homogeneous phase changes are directly integrated. In addition, a fitting correlation for the wall/vapor heat transfer coefficient is implemented and tuned against experimental data in order to evaluate the influence of the liquid film on conjugate heat transfer. The resulting numerical tool have been validated against experimental data achieved testing a copper Pulsating Heat Pipe during the $58^{\text {th }}$ ESA Parabolic Flight Campaign in several operative conditions and transient gravity levels. The predicted results show very good matching with the actual thermo-physical behavior of the system.
\end{abstract}

\section{KEYWORDS}

Pulsating Heat Pipe, Two-phase systems, Transient simulations, Microgravity, Lumped parameter model, Slug flow.

\section{INTRODUCTION}

Life and technology on Earth has evolved with a single environmental constant: gravity. Only in the last century, the advent of aeronautics and space flights set the focus on the effects caused by the alteration of the acceleration field, from the hyper-gravity experienced during lift off, to the microgravity perceived in orbit. Hyper-gravity conditions commonly arise in several ground applications too: cars and heavy vehicles may undergo hyper-gravity loads during sudden maneuvers. Any aircraft, civilian or military, can expose its components, payload and passengers to forces which excess $1 \mathrm{~g}$; this is especially true in military fighter jets and acrobatic aircrafts where the acceleration forces may be as high as $9 \mathrm{~g}$. In addition, enhanced gravity conditions may be encountered during centrifugal industrial processes, such as casting, material synthesis, even in chemical reactors. On the other hand, the so-called "microgravity condition" [1] exists in orbit, but it can also be reproduced on Earth: roller coaster on a dive, bungee jumpers and any objects in free fall can experience micro or reduced gravity conditions for few seconds.

The physical phenomena related to heat and mass transfer are greatly affected by the gravitational conditions and, for this reason, the current challenge is to design heat transfer devices able to operate efficiently under any acceleration condition. Being somewhat ubiquitous, thermal management can be found in electronics, energy management, transportation and in households in general. 
In the last decades, the industrial demand for higher performances, low cost and efficient thermal control has exponentially increased. Thus, a new generation of two-phase passive systems was born to satisfy the above general requirements. As a new and promising members of the heat pipes family, Pulsating Heat Pipes (PHPs) represent the last frontier of the two-phase passive heat transfer devices and could be an interesting alternative to standard cooling systems in the near future, having an extremely high potential in terms of simplicity and high heat transfer capability [2-3].

Essentially, a PHP consist of a tube, evacuated, partially filled with a working fluid and usually, but not necessarily, bended to arrange a closed serpentine [4-5]. Heat is provided in the so-called evaporator region and removed in the condenser zone. Since the channel is capillary, the main flow pattern recognizable in an operating PHP is the slug/plug flow even if this is not the only possible one [6]. In such conditions, an organized train of liquid slugs and vapor plugs surrounded by a thin liquid layer can be easily identified within the tube. The whole thermal-hydraulic behavior is complex. The fluidic batches can both circulate and oscillate driven by heterogeneous and homogeneous phase changes. Capillary forces and gravity may help or damp the fluid motion [714]. Simultaneously, liquid slugs and vapor plugs may change dimensions due to evaporation and condensation phenomena.

Since the late '90s researchers attempted to develop numerical tools in order to predict PHPs performance. Only few models are capable of providing complete thermal-hydraulic simulations and even fewer are partially validated against experimental data (see [15] and the recent review [16]). Existing models are usually very simple and contain many unrealistic assumptions (i.e. saturation conditions, constant liquid film). In addition, none is able to correctly represent the effects of transient states, start-up and dry out conditions. Nevertheless, validated numerical simulations can constitute useful tools to understand PHPs operational characteristics, and, in the future, to help the design of new and better performing ones.

Existing numerical models range from the continuum wave propagation approach [17-18] to the linearized mass-spring-dampers theory [19-21], from complex Artificial Neural Networks [22-24] to Computational Fluid Dynamics techniques [25-26]. Nevertheless, the most commonly adopted strategy is based on a sort of mono-dimensional lumped parameter approach. In this framework, the code developed by Holley and Faghri in 2005 [27] and later improved by Mameli et al. [28-30] represents a milestone in the PHP modelling. The authors assumed a priori saturated slug and plug flow and used a lagrangian approach to simulate the motion of the elements. The dynamics of the liquid film is neglected. The energy equation is implemented for both the fluidic and the solid domains. Heterogeneous phase changes are not directly accounted for in the mass balance even if liquid elements coalescence and new vapor formation are considered. Homogeneous evaporation and condensation through the interface, on the other hand, are totally neglected. In the latest version of the model [28-30], the effects of tube bandings on liquid slugs dynamics and the calculation of the two-phase heat transfer coefficient for liquid and vapour as a function of the different heating regime are included.

This paper proposes a revision of the above described models and aims to represent a step forward in the present PHPs modelling know-how. The common assumption of saturated vapor plugs has been removed; heterogeneous and homogeneous phase changes through the interface are directly integrated. In addition, since in the literature there is no empirical correlation of HTC for an oscillating vapour plug - liquid slug regime in the conditions of a PHP, a fitting correlation for the estimation of the wall/vapor heat transfer coefficient has been implemented and tuned against experimental values to evaluate the influence of the liquid film on the conjugate heat transfer.

An important effort has been devoted to correctly reproducing the effect of steady and transient gravity field on the PHP performances. Thus, the novel numerical tool has been validated in several operative conditions under various gravity levels. In the following, the comparison between simulated data and the experiments performed during the $58^{\text {th }}$ ESA Parabolic Flight Campaign is presented [12]. A copper PHP $(I D / O D=1.1 / 2.0 \mathrm{~mm})$ filled with FC-72, equipped with $14 \mathrm{~T}$ thermocouples and a pressure transducer has been tested providing different heat inputs (from 50W to $100 \mathrm{~W}$ ) at the evaporator side. The code shows a very good potentiality, reproducing with high accuracy the device transient operations during the simulated parabolic manoeuvres. 


\section{NUMERICAL MODEL}

Since the slug flow is the primary flow pattern in PHPs, this mono-dimensional, hybrid lumped parameters model assumes it a priori. However, as widely demonstrated by experiments and visualization studies [6], slug flow may not be the only flow regime occurring inside PHPs. Indeed the flow pattern may change into semi-annular or complete annular flow by increasing the heat flux input level. Thus, the present model is actually suitable for simulating PHPs working under relatively low heat fluxes.

In the following, three main sections describe respectively: the internal two-phase dynamics, the tube thermal behavior and, finally, the heat exchanged between the working fluid and the tube wall.

\subsection{Two-phase internal dynamics}

The PHP internal dynamics depends on the interplay between vapor and liquid. Since a confined flow is assumed, the fluidic elements are an alternation of $N_{j}$ liquid slugs and $N_{i}$ vapor plugs (Fig. 1); in addition, liquid slugs are subdivided into smaller sub-domains (liquid slices with equal length), having the same velocity, but different temperatures accounting for the axial heat conduction within the liquid phase. The number and the dimensions of all these fluidic batches are allowed to vary during the simulation in such a way that the total mass and length of the system are conserved.

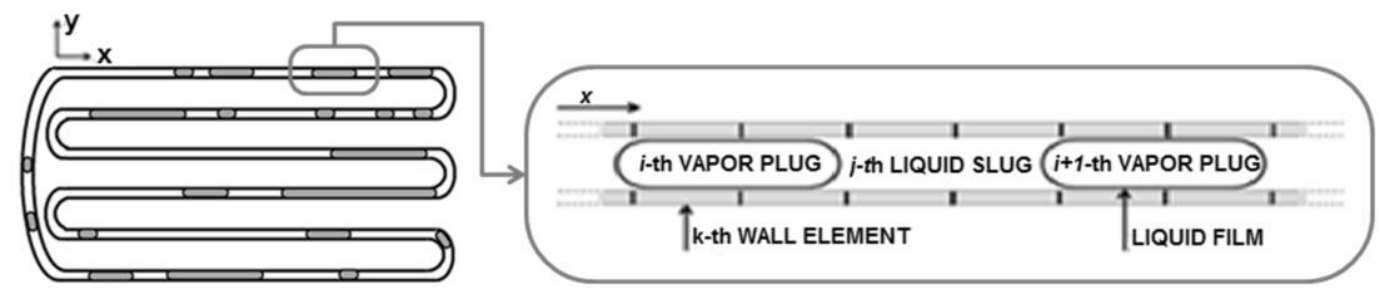

Fig. 1: Schematic of the numerical domains. The liquid slugs sub-domains are not explicitly indicated. The wall is considered completely wettable.

Mass, moment and energy balances are solved along the PHP axial direction under the following assumptions:

a. Slug flow is assumed a priori.

b. All the fluid thermo-physical properties, apart from vapor pressure and density, are function of the temperature only. Liquid is assumed incompressible.

c. The momentum equation for each liquid slug is lumped and friction between vapor plugs and wall elements is neglected.

d. Vapor is treated as a real gas (Van der Waals equation) except in case of phase changes. Density is calculated by its definition (mass over volume).

e. Vapor may exist in saturated, super-heated and sub-cooled conditions.

f. Heterogeneous phase changes are isothermal and isobaric; phase changes through the interface are isobaric but non-isothermal. Phase changes are always followed by isothermal compressions or expansions of vapor elements in order to re-adjust the total volume.

g. The temperature jump through the interface is neglected.

h. The momentum equation for each liquid slug is lumped; vapor/wall friction is neglected.

i. Liquid menisci maintain hemi-spherical shape with zero contact angles at the wall.

j. The thin liquid film around each vapor plug is neglected. It is only used to define the real volume occupied by each fluidic element within the tube inner channel, considering it constant in space and time.

\subsubsection{Mass balance and energy balance}

In PHPs, the local pressure disequilibrium, caused by phase change phenomena, represents one of the main driving forces at the base of the fluid motion. A fluidic element under condensation or 
evaporation varies its mass and, accordingly, dimension. However, the total mass and length of the PHP are constrained and must be conserved.

Both heterogeneous and homogenous phase changes through the interface may take place during PHP operation. Heterogeneous phase changes occur when a fluidic elements in saturated conditions comes into contact with a surface at different temperature involving heat transfer between the solid wall and the fluid. Thus a proper condition must be satisfied to allow heterogeneous phase changes, namely:

$\left\{\begin{array}{llll}p_{v}>p_{\text {sat }} & \& & T_{w} \leq T_{v, \text { sat }}-\Delta T_{\text {cooling }} & \text { for condensation } \\ p_{l}<p_{\text {sat }} & \& & T_{w} \geq T_{l, s a t}+\Delta T_{\text {super-heating }} & \text { for evaporation }\end{array}\right.$

where $p$ and $T$ stand respectively for pressure and temperature; the subscripts $v, l, w$ and sat refer to vapor, liquid, wall and saturated conditions respectively. $\Delta T_{\text {cooling }}$ and $\Delta T_{\text {super-heating }}$ are the temperature difference linked respectively to the nucleation onset and boiling nucleation.

Otherwise, if a fluidic element has a thermodynamic state deviating from saturation, homogeneous condensation or evaporation through the interface occurs in the fluidic bulk which represents the heat sink or source associated to this kind of transition.

$\left\{P_{v}>P_{\text {sat }}\right.$ for condensation

$\left\{P_{v}<P_{\text {sat }}\right.$ for evaporation

These phenomena occur simultaneously; however, in order to compute them, mass, momentum and energy balances are solved for liquid and vapor through a hierarchical method: for each time step, first the heterogeneous phase changes are solved, then the homogenous evaporation/ condensation phenomena through the interface are accounted for, finally all the other phenomena (e.g. sensible heat exchange with the wall, axial conduction, etc.) are computed. For more details refer to the attached annex A.

Note that $1^{\text {st }}$ order Adams-Bashforth integration scheme [31] has been adopted to integrate mass and energy balances if not differently indicated.

\section{Heterogeneous phase changes.}

Figure 2 shows in red the global control volume $(\mathrm{CV})$ adopted to solve mass and energy balances during heterogeneous condensation (A) and evaporation (B): it is closed, isochoric and diabatic with respect to the pipe walls. In case of condensation, it contains three sub-systems, one vapor $(V P)$ and two liquid elements $(L S)$ : the closed single vapor sub-domain allows a two-phase change and it is diabatic; the closed liquid sub-domains are mono-phase and adiabatic. The heterogeneous condensation will eventually occur in the vapor plug. In case of evaporation, $C V$ contains two vapor plugs and one liquid slug. Vapor sub-domains are closed, mono-phase and adiabatic, while liquid is closed and diabatic and allows two-phase changes. Thus, the heterogeneous evaporation will occur, eventually, in each liquid slice.

Accounting for the assumptions listed in the previous section (from $\mathrm{d}$ to $\mathrm{g}$ ), the integration of the mass and the energy balances applied to $C V$ and to each $L S$ or $V P$ (see the annex A) leads to the definition of the evaporated or condensed mass, $m_{L V, \text { het }}$ :

$m_{L V, h e t}=\left|\frac{A_{w f} q_{w f}}{h_{L V}}\right| \Delta t$

where $A_{w f} q_{w f}$ is the heat transferred to or from the wall (section 2.3), $h_{L V}$ the heat of vaporization and $\Delta t$ the computational time step. 


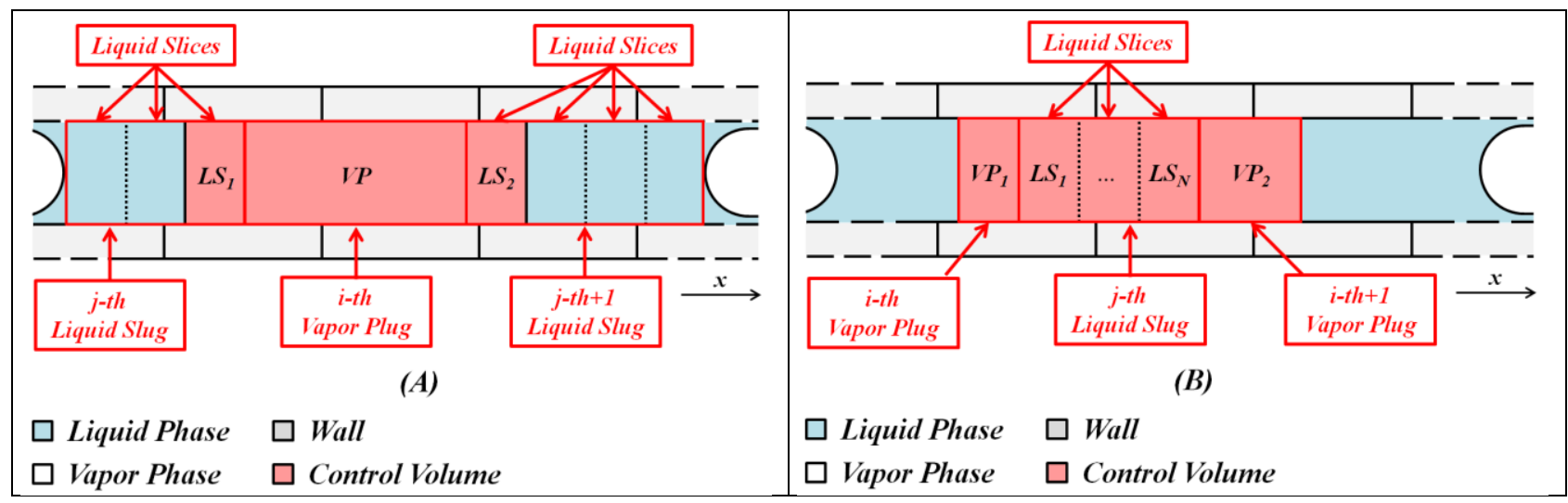

Fig. 2: Schematic of the fluidic control volumes CV (in red) adopted in case of heterogeneous condensation (A) or evaporation (B).

Note that heterogeneous phase changes may induce the formation of new fluidic elements, as well as the dimension rescaling of already existing liquid slugs and vapor plugs. Specifically, a new element is produced only if the evaporated or condensed mass, $m_{L V, h e t}$, respects the assumption of confined flow, $m_{L V \text {,het }} \geq m_{\min }$ where the minimum value is computed as the mass of an equivalent sphere which fits perfectly within the tube $\left(m_{\text {min,eva(cond })}=\frac{1}{6} \pi d_{i n}^{3} \rho_{v(l)}\right)$. In addition phase changes may cause the disappearance of small fluidic domains and the merging of adjacent slugs/plugs if $m_{L V \text {,het }}$ exceeds the mass of the corresponding element.

\section{Homogeneous phase changes.}

Heterogeneous phase changes are computed for all the fluidic elements. Only at this point, on the new fluidic path, evaporation and condensation through the interface can take place $\left(2^{\text {nd }}\right.$ phase of the hierarchical numerical procedure). The adopted control volume $C V$ is shown in Fig. $2 \mathrm{~A}$; It is considered closed, adiabatic and isochoric. The two liquid slices and the vapor plug are open and adiabatic. However, if the control volume is adiabatic, it cannot be assumed that the phase changes are both isothermal and isobaric, thus only the assumption of $d P=0$ has been maintained (assumption $\mathrm{f}$ ).

Accounting for conservation of mass and volume (Fig. 3), a linear systems of equations (Eq. 4) allows estimating the evaporated and condensed mass, $m_{L V, h o m}$, and the final dimension, $V_{v, t+1}$, of the involved vapor element (see Annex A).

\section{Evaporation}

$\left\{\begin{array}{l}m_{L V, h o m}=\left|\Delta m_{v}\right|_{t_{i}}^{t_{i+1}} \mid \\ V_{v, t+1}=V_{v, t}+\frac{m_{L V, h o m}}{\rho_{l}}\end{array}\right.$

\section{Condensation}

$$
\left\{\begin{array}{l}
m_{L V, h o m}=\left|\Delta m_{v}\right|_{t_{i}}^{t_{i+1}} \mid \\
V_{v, t+1}=V_{v, t}-\frac{m_{L V, h o m}}{\rho_{l}}
\end{array}\right.
$$

where $V$ stands for volume and $\rho$ for density.

In addition, homogeneous phase changes through the interface occurs in the fluidic bulk which represents, indeed, the heat sink or source associated with this kind of transition: in particular a liquid which undergoes evaporation cools down, while vapor that is condensing undergoes a heating process:

\section{Evaporation}

$\left.m_{l, t_{i}} \Delta u_{l}\right|_{t_{i}} ^{t_{i}+1}=-h_{L V} m_{L V, h o m}$

\section{Condensation}

$$
\left.m_{v, t_{i}} \Delta u_{v}\right|_{t_{i}} ^{t_{i}+1}+\left.R^{*} m_{v, t_{i}} \Delta T_{v}\right|_{t_{i}} ^{t_{i}+1}=h_{L V} m_{L V, h o m}
$$

where $u$ is the specific internal energy of the involved fluidic elements. 


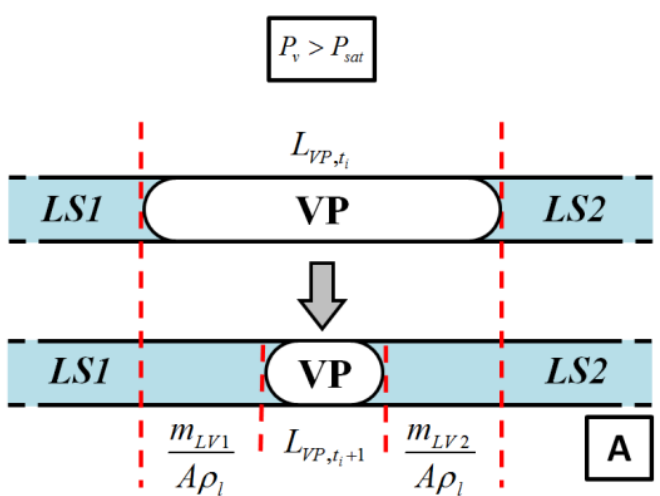

Liquid Phase $\square$ Vapor Phase

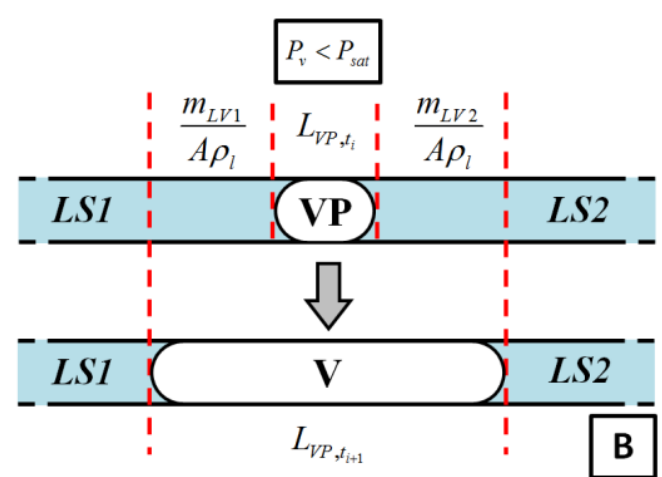

Liquid Phase $\square$ Vapor Phase

Fig. 3: Schematic of homogeneous condensation (A) and evaporation (B). Since the cross sectional area is constant, $V=A L$.

\section{Closing procedure after the phase change intermediate step.}

In order to complete mass and energy balances for each time step, after having taken into account heterogeneous phase changes and homogeneous evaporation/condensation through the interface, all other physical phenomena (e.g. sensible heat exchange with the wall, axial conduction, etc.) should be considered. Note that the three steps of this procedure are occurring in sequence during the same time step $\Delta t$. The control volumes adopted this time consist of single vapor plugs or single liquid slices (see Fig. 4) representing closed domains. A Lagrangian approach has been adopted, thus one temperature is calculated for each fluidic element. Nevertheless, in order to account for the conduction within liquid slugs, increasing the resolution of the energy field, liquid slugs are equally subdivided into smaller sub-domains; conduction within vapor is, on the contrary, negligible because of the small conductivity.

In a Lagrangian system, total time derivatives match ordinary time derivatives; thus, assuming constant specific heat $c_{V}$, the energy equation is:

$$
c_{V, f} m_{f, n} \frac{d T_{f, n}}{d t}=\left.\left(q_{w f, f} A_{w f}\right)\right|_{n}+\left(\left.k_{f} A \frac{\partial T_{f}}{\partial x}\right|_{n} ^{n-1}-\left.k_{f} A \frac{\partial T_{f}}{\partial x}\right|_{n+1} ^{n}\right)-P_{f, n} \frac{d V_{f, n}}{d t}
$$

where the subscript $f$ stands for fluidic, alternatively vapor or liquid; $\mathrm{A}$ is the cross sectional area (Fig. 4) while $k$ is the thermal conductivity .

The first term on the right hand side of Eq. 6 accounts for sensible heat transferred between the wall and the fluid when no phase changes have occurred previously; the second term is the axial conduction within the fluid (for the subscripts refer to Fig. 4); the last term is the compression work computed only for vapor plugs.

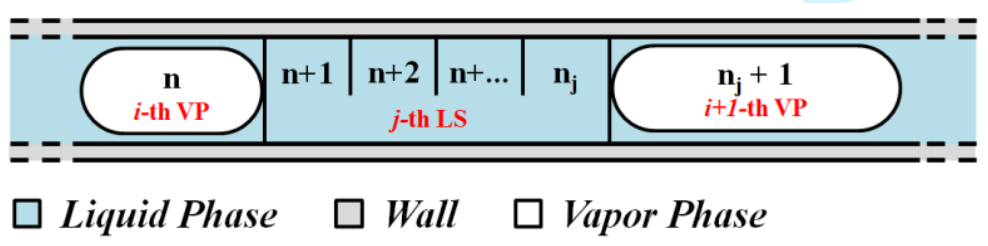

Fig. 4: Schematic of the fluidic domains and sub-domains accounted for the energy balance computation. VP stands for vapor plug, LS stands for liquid slug.

A $1^{\text {st }}$ ordr Adams-Bashforth scheme is applied for the integration of the energy equation if elements were born or disappeared in the time step; otherwise, the $2^{\text {nd }}$ order Adams-Bashforth scheme is used [31]. 


\subsubsection{Momentum Equation}

Liquid slugs and vapor plugs move within the PHP channel. Their motion is simultaneously oscillatory and circulatory. A Lagrangian approach simplifies the traceability of all the fluidic elements; the Störmer-Verlet algorithm, in its velocity variant, is chosen for the computation of velocities and positions due to its well known ability to capture the long term system dynamics in an accurate and stable way [32]. The momentum equation has been solved directly for the global time step $\Delta t$ without accounting separately for phase changes; thus $d m \neq 0$ if evaporation or condensation has occurred; however it is a known quantity for all the liquid slugs.

Vapor/wall friction is neglected (assumption $\mathrm{h}$ ), thus the momentum equation can be evaluated only for liquid elements imposing that vapor is dragged, consequently, along the tube. A first integration of the momentum equation along the $j$-th liquid slug provides the velocity, $w$ (Eq. 7); a second integration defines the position, $x$ (Eq. 8).

$\frac{d\left(m_{l} w\right)}{d t}=m_{l} g \cos (\vartheta)+A\left(p_{i}-p_{i+1}\right)-0.5 f_{\tau} \frac{m_{l}}{d_{\text {in }}} w^{2}$

$x_{t}=x_{t-1}+w \Delta t+\frac{a}{2} \Delta t^{2}$

$d_{\text {in }}$ is the tube internal diameter; $a$ is acceleration acting of the $j$-th liquid element defined as the ratio between the applied forces and mass.

The first term on the right side of Eq. 7 represents the gravity force: $g$ is the gravity acceleration and $\vartheta$ is the local angle between the gravity vector and the flow direction. The second and the third terms are the forces respectively due to adjacent vapor expansion/compression (for the subscripts refer to Fig. 4) and friction (viscous shear and minor losses due to bends and turns), which is treated semi-empirically. The shear friction coefficient $f_{\tau}$ is evaluated making use of the Hagen-Poiseuille equation [33] (fully developed laminar flow) or the Haaland equation [34] (turbulent flow). As suggested by Mameli et al. [29], local losses are computed only if the liquid slug passes through at least a turn or a bend; the corresponding friction coefficient is evaluated according to Darby $3 \mathrm{~K}$ method [35]. Capillary forces are neglected because of the constant cross section along the tube length and because liquid menisci maintain spherical shape with zero contact angle at the wall (assumption i).

\subsection{Wall energy balance}

The PHP tube thermal behavior can be deduced integrating the wall energy balance. $N_{w}$ eulerian domains are defined and equally split the channel wall (Fig. 1). The associated masses and positions are fixed.

Under the assumption of constant thermal properties, the variation of the $k$-th wall temperature in time is:

$m_{w} c_{V, w} \frac{d T_{w}}{d t}=\left(\left.k_{w} A_{w} \frac{\partial T_{w}}{\partial x}\right|_{k} ^{k-1}-\left.k_{w} A_{w} \frac{\partial T_{w}}{\partial x}\right|_{k+1} ^{k}\right)-q_{w f, w} A_{w f}+q_{e x} A_{e x}$

$A_{e x}$ is the external tube surface exposed to the environment; $A_{w}$ is the tube cross section area.

The first term on the right side of Eq. 9 accounts for the heat conduction within the wall; the second term is the heat exchanged between the wall and the fluidic elements; the last term represents the heat exchanged between the wall and the external environment. Constant heat input power $Q_{e x}$ is supplied to the evaporator zone, forced convection is applied at the condenser, while no heat exchange occurs in the adiabatic region. Radiation has been neglected being less than $0.5 \%$ of the total dissipated heat in the worst case. 
$q_{\text {ex }} \begin{cases}Q_{\text {ex }} / A_{\text {ex }} & \text { evaporator zone } \\ 0 & \text { adiabatic zone } \\ h_{\infty}\left(T_{\infty}-T_{w}\right) & \text { condenser zone }\end{cases}$

$h_{\infty}$ is the external heat transfer coefficient; $T_{\infty}$ the environmental temperature.

The $1^{\text {st }}$ order Adams-Bashforth scheme is applied for the integration of the wall energy equation.

\subsection{Solid/Fluid Coupling}

The joining link between the wall and the fluidic domains is the heat exchanged in radial direction, $q_{w f}$, which appears in the energy balances (sections 2.1.1 and 2.2.

The wall domains are fixed in time, the fluidic elements are moving. Thus, a fluidic element may face different wall domains at each time step; in addition a wall element may face more than one fluidic element and vice versa (Fig. 5). Thus, accounting for an appropriate heat transfer coefficient $h, q_{w f}$ is defined as:

$$
\left\{\begin{array}{l}
q_{w f, w}=\frac{1}{A_{w f}} \sum_{n=1}^{N_{f}} h\left(T_{w}-T_{f, n}\right) A_{w f, n} \\
q_{w f, f}=\frac{1}{A_{w f}} \sum_{k=1}^{N_{w}} h\left(T_{w, k}-T_{f}\right) A_{w f, k}
\end{array}\right.
$$

$A_{w f}$ is the total area between the wall and the fluidic domains; $A_{w f, n}$ and $A_{w f, k}$ represent the area in common between the $k$-th wall element and the $n$-th fluidic element.

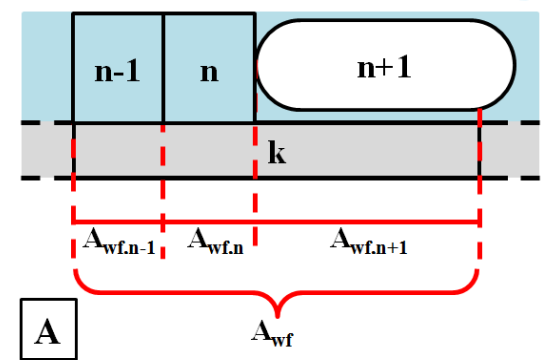

Liquid Phase
Wall

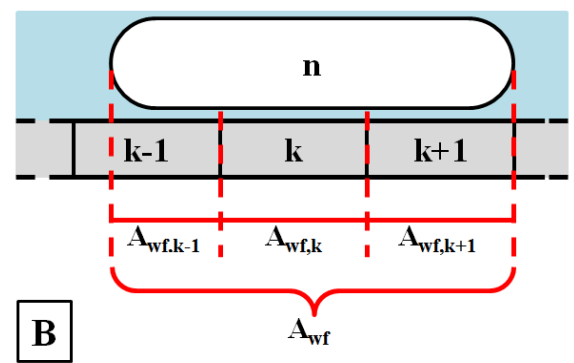

Vapor Phase

Fig. 5: Definition of $A_{w f}, A_{w f, n}$ and $A_{w f, k}$ from the wall point of view (A) and from the fluid point of view (B).

The heat transfer coefficient should account for sensible or latent heat, depending on if phase changes are occurring or not during the time step lag.

\section{Sensible heat transfer coefficient.}

In the case of liquid slugs, sensible heat transfer is calculated with semi-empirical formula, even if these are not strictly applicable to oscillating flows in mini-channels. Shah and London [36] correlation, Gnielinski correlation [33] and Dittus-Boelter correlation [33] are implemented respectively for the laminar flow thermally developing region, for the transient/turbulent flow, and for the fully developed turbulent flow.

The sensible heat exchanged between wall and vapor, on the other hand, cannot be estimated by means of the above reported classical correlations. The reason lies in the presence of the liquid film. First, the model considers its thickness constant in space and time (assumption j). Actually, the film dynamic may deeply influence the heat transferred by vapor plugs [37-38]. Thus a fitting correlation has been proposed to fix the lack of modelling. Accounting for both forced and natural convection and making use of the Buckingham theorem, the vapor-wall sensible heat transfer coefficient is 
1307

derived as a function of both Reynolds $(R e)$ and Rayleigh $(R a)$ numbers. The four fitting coefficients are estimated by comparison with experimental results in different operative conditions and under various gravity levels.

$$
\begin{array}{rlrl}
h=h_{0}+a R e^{b} R a^{c} & h_{0}=200 \frac{W}{m^{2} K} & b=0.25 \\
a & =70 \frac{W}{m^{2} K} & c & =0.4
\end{array}
$$

$a$ is defined at $293 \mathrm{~K}$ for a conventional film thickness of $50 \mu \mathrm{m}$ [39].

Phase change heat transfer coefficients.

Constant values are assumed, since experimental correlations are missing.

$h= \begin{cases}10000 \frac{W}{m^{2} K} & \text { condensation } \\ 20000 \frac{W}{m^{2} K} & \text { evaporation }\end{cases}$

A sensitivity analysis excluded a strong influence of the imposed values on the model results: the maximum steady state temperature deviation was less than $0.5 \mathrm{~K}$ for evaporating/condensing heat transfer coefficients ranging from $4000 / 2000 \mathrm{~W} / \mathrm{m}^{2} \mathrm{~K}$ [5], to $30000 / 15000 \mathrm{~W} / \mathrm{m}^{2} \mathrm{~K}$. This because most of the heat is transferred by sensible ways only, as underlined by Shafii et al. [40], the latent heat associated to phase change is prevalently the driving motors of the system inducing oscillation/circulation of the fluid within the channel.

\subsection{Numerical time step}

The computational time step is generally constant, but, when an element is generated or disappear, $\Delta t$ is reduced by one order of magnitude in order to damp any possible numerical unstable oscillation; after 10 iterations, it is gradually restored following a slope increment. A sensitivity analysis emphasized that, once the time step is small enough to guarantee numerical stability, the results are independent on the chosen value. For the tested conditions (Section 3) a $\Delta t$ of $0.4 \mathrm{~ms}$ is the maximum allowable and, thus, it is chosen to minimize the total simulation time.

\section{PARABOLIC FLIGHT CAMPAIGN}

The thermal response of a PHP is strongly influenced by gravity [7-14], as can be easily inferred from the provided supplementary videos showing the numerical simulated motion of the fluidic elements within the PHP channel when different levels of gravitational field are applied $(-1,0,1 \mathrm{~g})$. During the $58^{\text {th }}$ Parabolic Flight Campaign several experimental tests have been performed on a planar, bottom heated mode PHP partially filled with FC-72 under different operative conditions [12]; specifically, six different power levels have been analyzed, from $50 \mathrm{~W}$ to $100 \mathrm{~W}$ with step of 10W. A parabolic flight allows to test a device under various gravity levels. During each flight, indeed, the plane performs thirty-one parabolic manoeuvres which are subdivided into three parts: $20 \mathrm{~s}$ at $1.8 \mathrm{~g}$ (hyper-gravity) followed by $22 \mathrm{~s}$ at $0.01 \mathrm{~g}$ (micro-gravity) followed again by $20 \mathrm{~s}$ at $1.8 \mathrm{~g}$. A 90s pause of normal gravity is kept before and after each parabola.

The developed lumped parameter model has been applied to simulate these parabolic tests. The numerical results match with the experimental data, in term of steady state conditions as well as transient time.

Table 1 lists both the geometrical characteristic of the tested PHP and the numerical input of the code.

Basically, the PHP structure consists of a copper channel (D.I. $1.1 \mathrm{~mm} / \mathrm{D} . \mathrm{O}$. $2.0 \mathrm{~mm}$ ) folded so as to obtain 32 parallel branches. The tube is evacuated and partially filled of degassed FC-72 (0.50 volumetric filling ratio). The PHP is heated up making use of electric resistors wrapped around the 
evaporator bends, while the condenser section is embedded into a heat sink and cooled by forced convection. A pressure transducer is plugged outside the condenser section; an accelerometer tracks the gravity load during the flight maneuvers; in addition, the PHP is equipped with calibrated T thermocouples, nine located few millimetres above the evaporator zone, four in the condenser region and one is measuring the external temperature.

Since the experimental evaporator thermocouples are not exactly located in the hot section, but just above it in the adiabatic zone because of the presence of the heating wire, in order to be coherent with the model described in the previous section, the temperatures recorded by these sensors will be compared with the ones computed in the numerical adiabatic region. Therefore, experimental evaporator temperatures are referred in the following as adiabatic temperatures.

The results are presented mainly in terms of local spatial average temperature evolutions plotted together with the gravity level (as fraction of conventional $g$ on Earth) on the secondary y-axis. Since the experimental results are repeatable, only one parabola for each condition is shown. The graph on the left represents the experimental data, while the diagrams on the right report the numerical results. The grey solid line marks the temperature in the adiabatic zone, the blue line characterizes the condenser region and the green line indicates the temperature of the external environment. The numerical graphs report also a red line for the mean temperature in the evaporator region. The light colored regions on the experimental temperatures represent the standard deviation. An additional dynamic comparison for the $50 \mathrm{~W}$ parabola is provided as supplementary material.
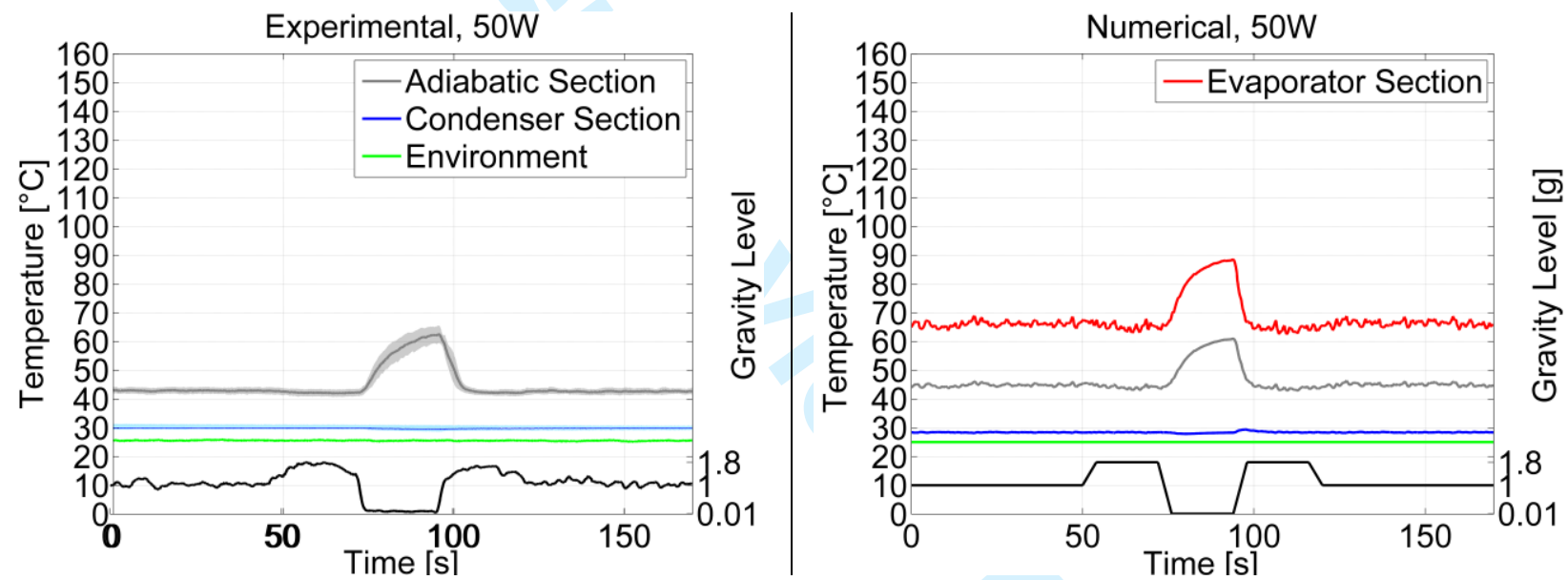

Fig. 6: Experimental and numerical evolution of the local spatial average temperatures for a bottom heated mode PHP at 50W. Solid lines represent mean temperatures; colored regions indicate the standard deviation on the recorder data.
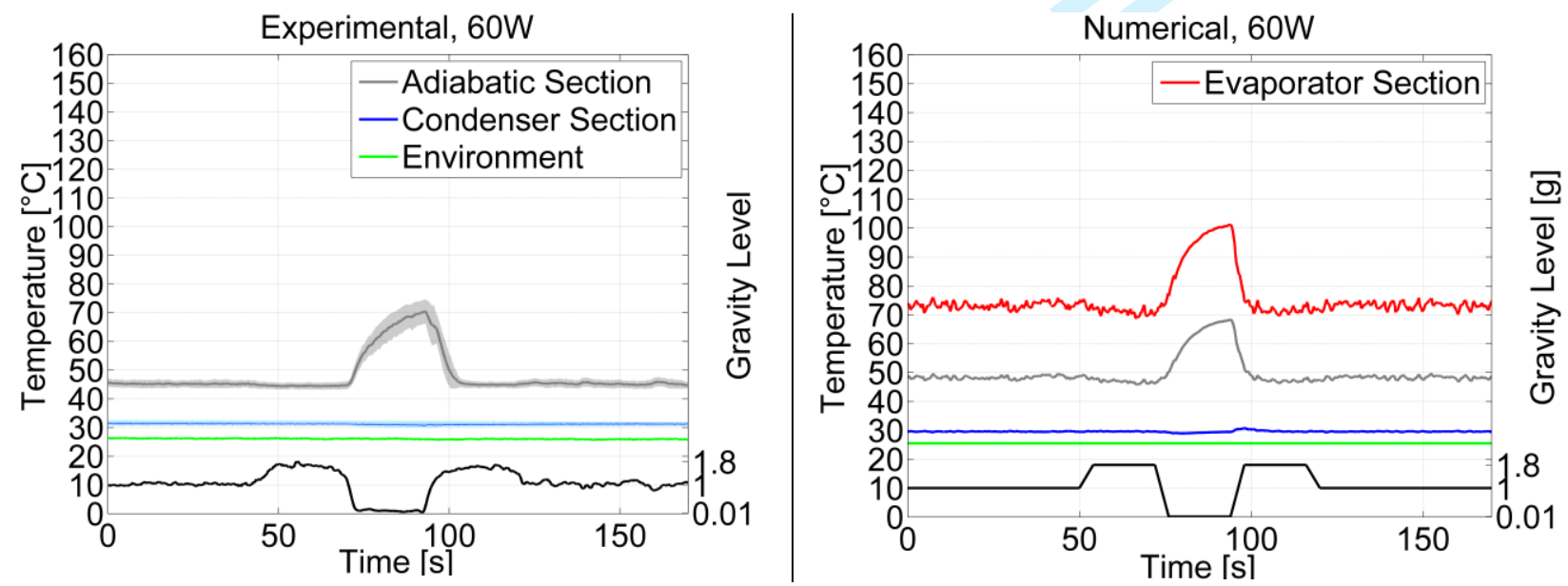

Fig. 7: Experimental and numerical evolution of the local spatial average temperatures for a bottom heated mode PHP at $60 \mathrm{~W}$. Solid lines represent mean temperatures; colored regions indicate the standard deviation on the recorder data. 

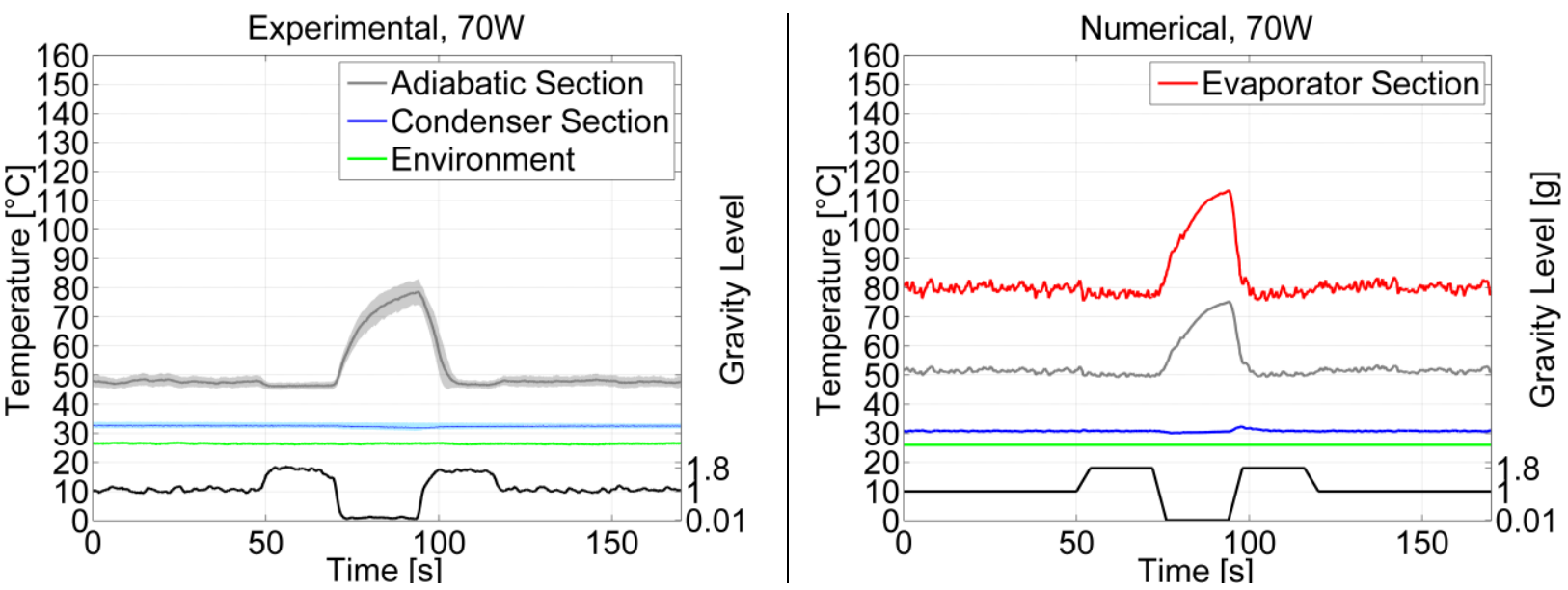

Fig. 8: Experimental and numerical evolution of the local spatial average temperatures for a bottom heated mode PHP at 70W. Solid lines represent mean temperatures; colored regions indicate the standard deviation on the recorder data.
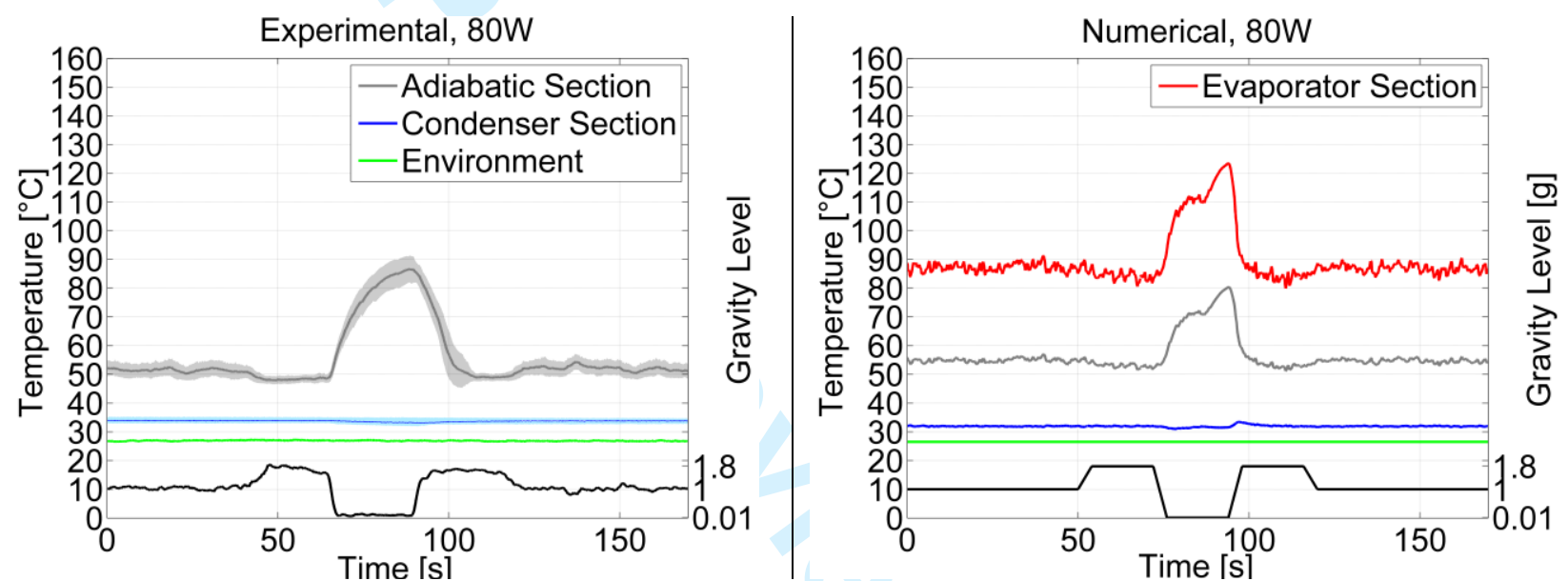

Fig. 9: Experimental and numerical evolution of the local spatial average temperatures for a bottom heated mode PHP at $80 \mathrm{~W}$. Solid lines represent mean temperatures; colored regions indicate the standard deviation on the recorder data.
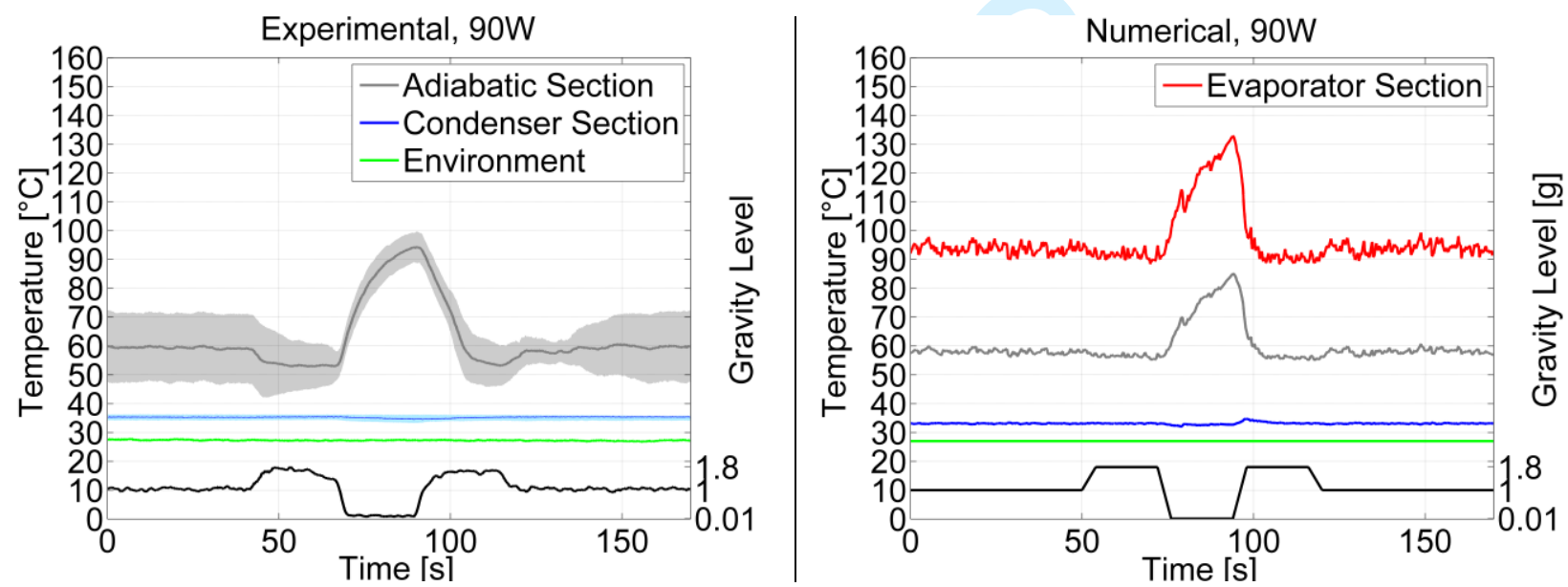

Fig. 10: Experimental and numerical evolution of the local spatial average temperatures for a bottom heated mode PHP at $90 \mathrm{~W}$. Solid lines represent mean temperatures; colored regions indicate the standard deviation on the recorder data. 

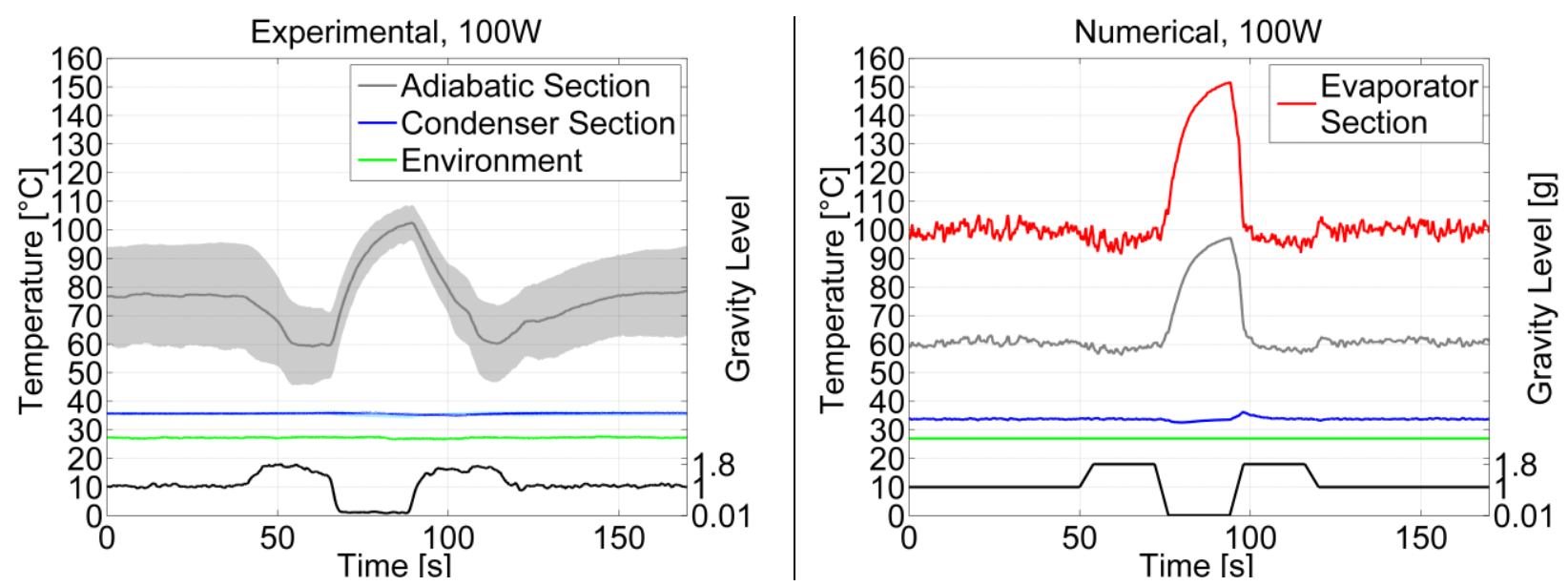

Fig. 11: Experimental and numerical evolution of the local spatial average temperatures for a bottom heated mode PHP at $100 \mathrm{~W}$. Solid lines represent mean temperatures; colored regions indicate the standard deviation on the recorder data.

The reported comparisons (Fig. 6-9) show the high accuracy of the numerical model both in steady and in transient conditions. The temperatures in all the PHP regions are correctly evaluated in terms of mean values, as well as in terms of temporal evolution for all the gravity levels and powers tested (from $50 \mathrm{~W}$ to $80 \mathrm{~W}$ ).

At $90 \mathrm{~W}$ and 100W (Fig. 10 and Fig. 11), on the other hand, the error on the mean temperature is increased, even if the temporal evolution is followed. The reason of that lies in the liquid film dynamics. Experimentally, at high power levels, some PHP branches undergo dry-out, worsening the whole device performances. Figure 12 shows an example of this unstable condition reporting all the recorded temperatures for the 100W parabola: dry-out is clearly visible in the most part of the PHP branches.

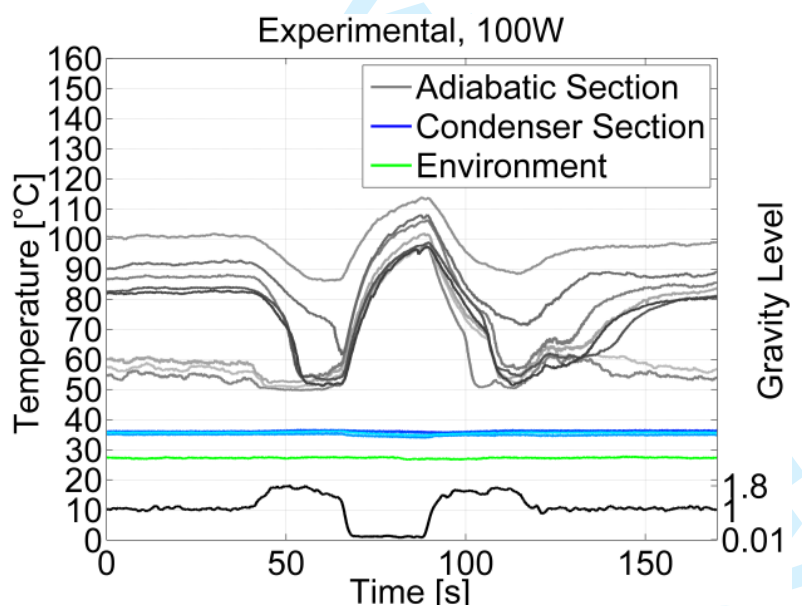

Fig. 12: Experimental wall temperatures for a bottom heated mode PHP at 100W. Greyish colours represent the adiabatic zone, bluish indicate the condenser section.

This sudden thermal crisis is originated by local complete evaporation of the liquid film and consequently reduction of the vapor/wall heat transfer coefficient in the corresponding regions. This instability reduces at high g-levels, since the boosted gravity force slightly improves the whole PHP performances when a bottom heated mode configuration is accounted for [11]. The circulation of the fluid within the channel, indeed, is promoted because liquid slugs are energetically pushed down from the condenser back to the evaporator section; in addition the enhanced gravity induce a downwards motion of the liquid film, creating a sort of small pool in the hotter region and, thus, reducing the possibility of local dry-out. Since, the model assumes constant film thickness (assumption $\mathrm{j}$ ), this kind of behaviour cannot be detected and, therefore, for this reason, the simulated temperature may be lower than the experimental data (e.g. $1 \mathrm{~g}$ at $100 \mathrm{~W}$ ); at the same time, the numerical temperature lowering at $1.8 \mathrm{~g}$ may be smaller than the real ones (e.g. $1.8 \mathrm{~g}$ at $90 \mathrm{~W}$ ). In 
1414 415 416

\section{5}

addition, since slug flow is assumed a priori, the code is not able to model the flow pattern transition in case of high heat flux levels.
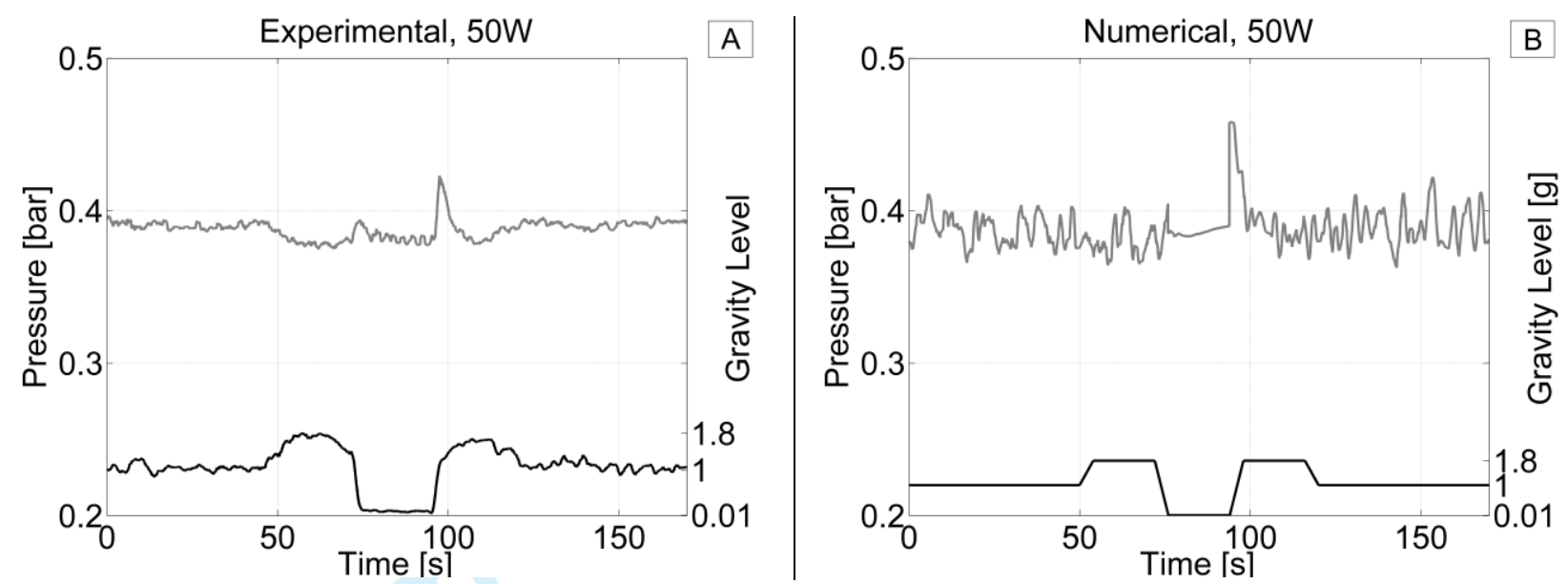

Fig. 13: Experimental (A) and numerical (B) pressure signal for the 50W parabola.

The experimental results show that both the occurring of the hyper and the micro-gravity conditions affect the PHP thermal response. Since the gravity vector is parallel to the flow path and oriented from the condenser to the evaporator zone, it gives a net contribution to the fluid momentum. The effect of micro-gravity is evident for all the heat input levels: temperatures in the evaporator increase because the fluid motion is no more assisted by gravity, pressure oscillations are less frequent (see, for example, Fig. 13a for the PHP at 50W) and consequently the heat transfer rate is less efficient. The occurring of the second hyper-gravity period assists the fluid motion bringing back the device to the previous thermal regime.

This explanation is also confirmed by the numerical results. Figure $13 \mathrm{~b}$ shows the fluid pressure for the PHP simulated at 50W. Similar results have been achieved for the other power levels. Differently from the experimental data recorded in one point outside the condenser section, the reported pressure is a mean value over the fluid elements in the whole condenser region (because of the Lagrangian approach). Although the pressure signal is damped with respect to the experimental one, in both cases the oscillations of the signal reduce when micro-gravity conditions are established; then they are restored with a high isolated pick when $1.8 \mathrm{~g}$ are applied on the PHP again. It is worth to note that the internal motion does not stop during these 20s: Fig. 14 shows the velocity of the liquid elements within the channel. During the micro-gravity phase, the velocity suddenly decreases but small oscillations are still present.

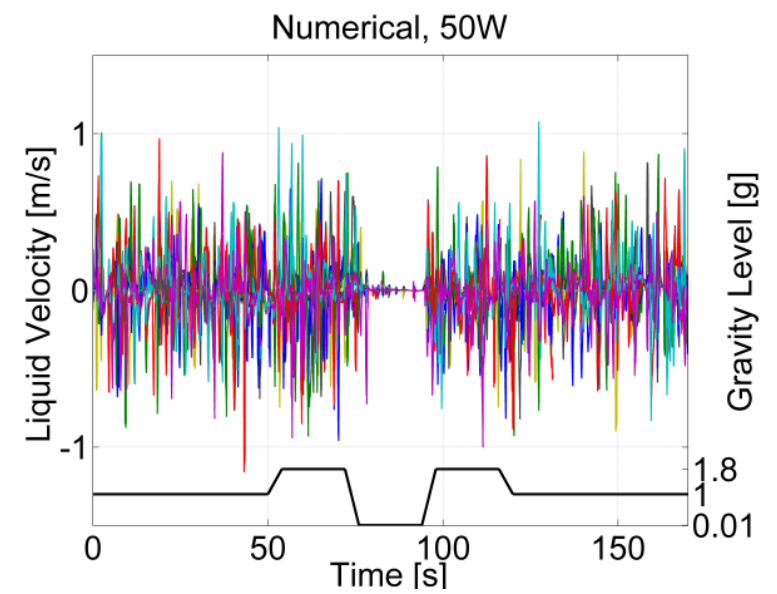

Fig. 14: Numerical computed liquid slugs velocities for the $50 \mathrm{~W}$ parabola. Different colours indicate different elements.

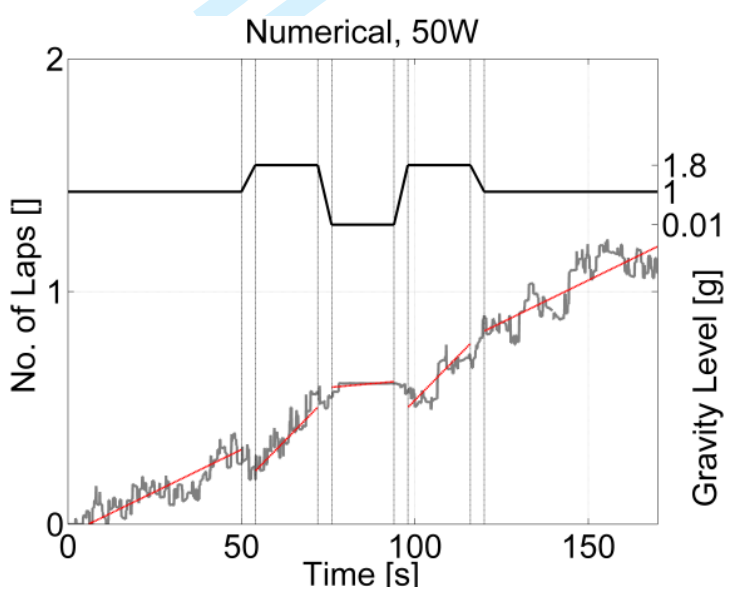

Fig. 15: 50W parabola; number of fluidic laps. Red dotted lines report the linear interpolation curves. 
1439

440

Liquid elements move within the micro-channel. The total velocities reported in Fig. 14 account for both oscillation and circulation. During the micro-gravity phase, only the circulating velocity reduces to zero, while the oscillating one is still present even if it is strongly reduced. This can be easily inferred from Fig. 15. It shows the number of laps performed, on average, by the fluidic elements during the simulation time. The slope associated to each interpolating red curve represents the mean circulation frequency of the fluid in the analyzed conditions. The fluctuations on the gray curve underline that the fluid is strongly oscillating when gravity is acting. As expected the circulation frequency, and thus the circulation velocity, increases when passing from $1 \mathrm{~g}$ to $1.8 \mathrm{~g}$ and from $0.01 \mathrm{~g}$ to $1.8 \mathrm{~g}$. This enhances the PHP performances. During the micro-gravity period, instead, the circulation of the fluid stops, being the red curve horizontal.

\subsection{Ground tilting tests}

Mameli et al. [12] reported that the dynamic response of the PHP during a parabola test is comparable with the behavior of the PHP on ground during the a "Vertical to Horizontal to Vertical" maneuver. The maximum temperatures reached during micro-gravity and during the horizontal period on ground were, indeed, comparable for all the heat input levels. The same happens when this tilting "Vertical to Horizontal to Vertical" maneuver is numerically simulated, see, for example, Fig. 16 corresponding to the $50 \mathrm{~W}$ test.
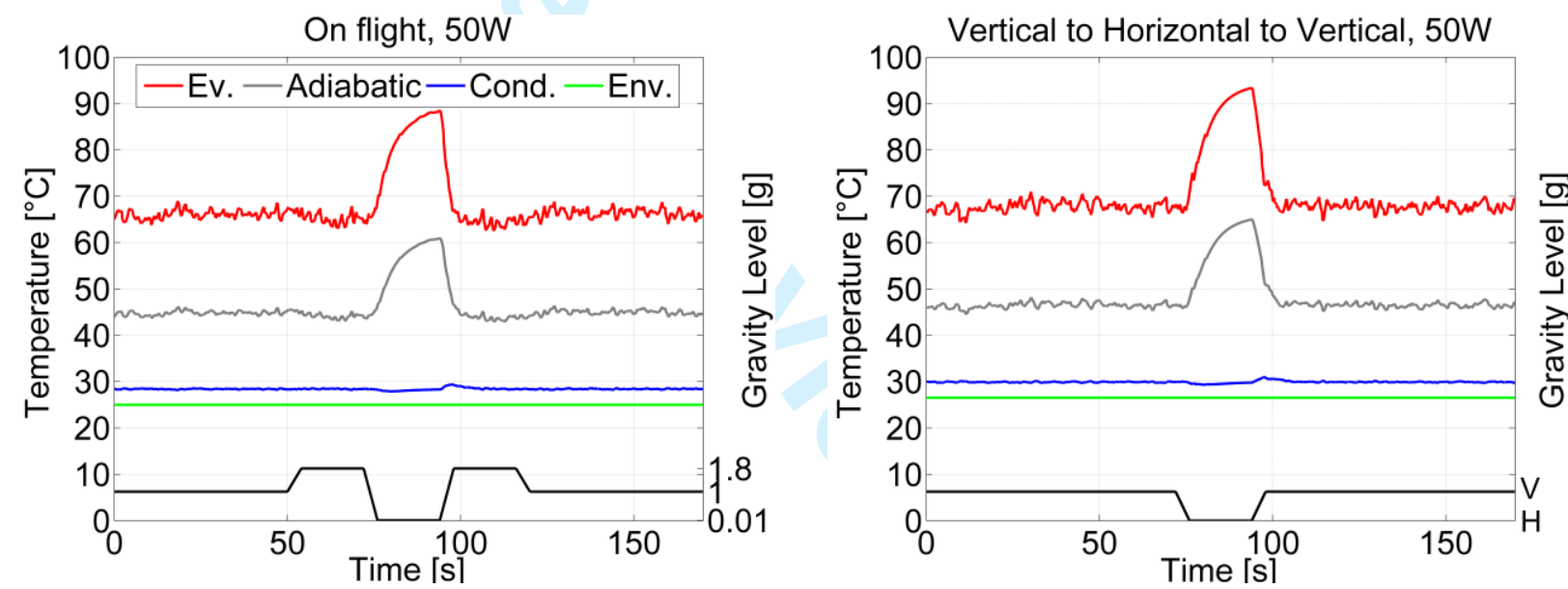

Fig. 16: Numerical evolution of the local spatial average temperatures for the $50 \mathrm{~W}$ parabola (on the left) and the 50W tilting manoeuvre (on the right).

The numerical simulations, as well as the experimental test, observe a small temperature increment during the micro-gravity phase for the ground tests with respect to the flight trials. In the reported case of $50 \mathrm{~W}$ this deviation is less than $4 \%$. However this temperature gap is easily explainable: the lack of the first hyper-gravity phase fastens the degradation of the PHP performance during the 20s of micro-gravity.

In addition, as experimentally observed, due to the lack of the second hyper-gravity phase, the device is not able to reach the previous thermal regime as fast as during the flight. This happen at all the power levels, however it is more evident for high heat input values. The comparison of the results achieved at $70 \mathrm{~W}$ is reported here as an example to support this last statement (Fig. 17). 

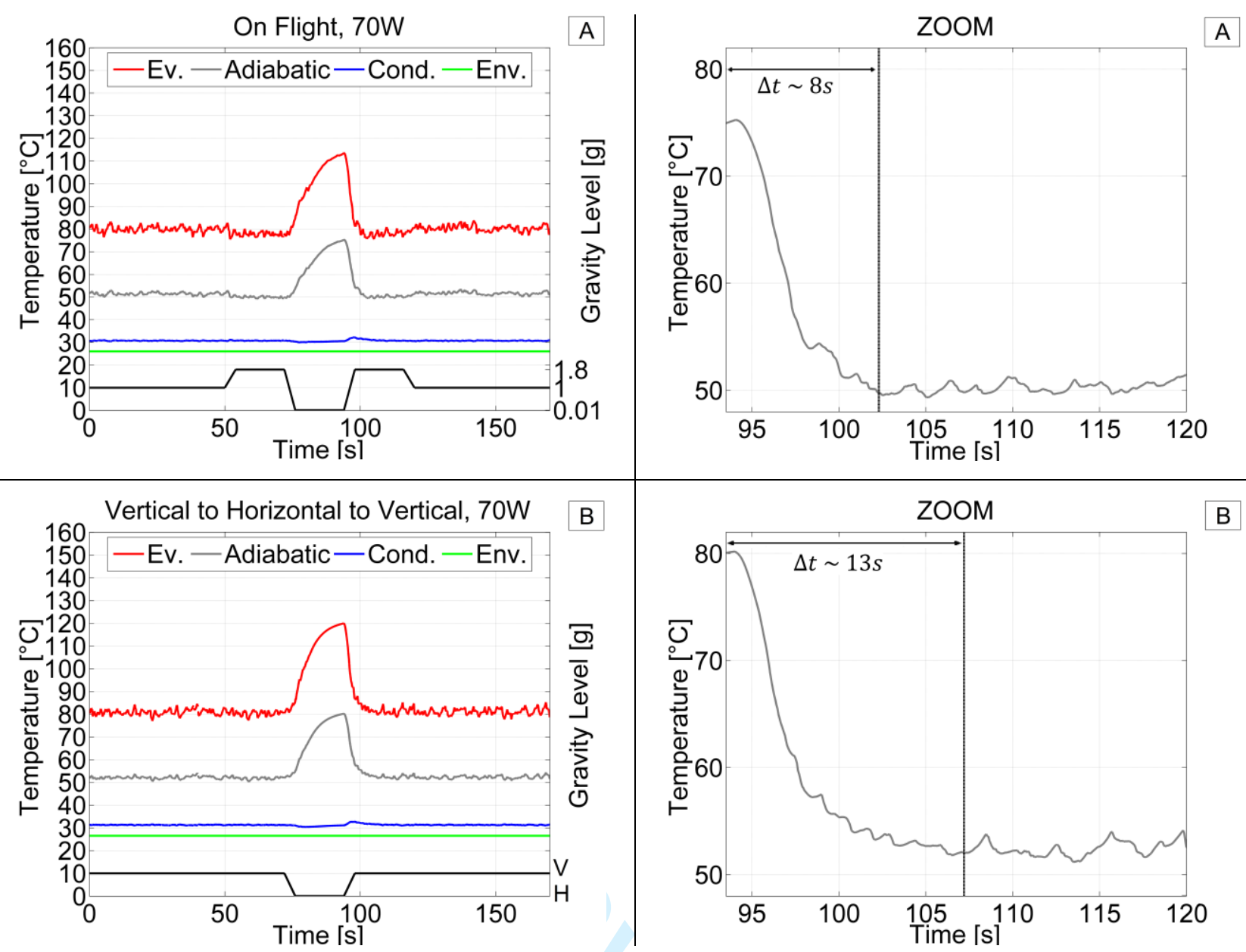

Fig. 17: Numerical evolution of the local spatial average temperatures for the 70W parabola (A) and the 70W tilting manoeuvre $(B)$. On the right, zoom of the adiabatic temperature in the transition zone.

\section{CONCLUSIONS}

The thermal performances of a bottom heated mode PHP tested during the $58^{\text {th }}$ ESA Parabolic Flight Campaign have been simulated with a novel one dimensional hybrid lumped parameter code. The comparison between experimental and numerical results highlights the high prediction capability of the model.

The numerical code is able to reproduce with high accuracy both the stationary values and the transient evolution of the local spatial average temperature that a PHP experiences during a parabolic flight. In addition, the internal dynamic is qualitatively predicted.

During the reduced gravity period, as experimentally proved, the simulated temperatures in the evaporator region increase because the fluid motion is no more assisted in its flowing back from the condenser to the evaporator region. The pressure oscillations are less frequent, the fluid stops its circulation even if oscillations are still present; the heat transfer is, therefore, less efficient. On the other hand, the hyper-gravity phases, slightly improve the device thermal performance: the numerical simulations, indeed, record higher circulation frequency. In addition the second hypergravity phase speed up the recovery of the thermal performances after the $20 \mathrm{~s}$ at $0.01 \mathrm{~g}$. This is confirmed by ground tests achieved with a "Vertical to Horizontal to Vertical" operation. Such kind of experiments, indeed, are totally comparable with the flight trials except the lack of the hypergravity phases.

Unfortunately, the model is not able to account for the liquid film dynamic. Dry-out appears when strong evaporation phenomena locally generates disappearance of the liquid layer. This generally happens at high power levels, over $90 \mathrm{~W}$ for the analysed case. The assumption of constant film thickness, therefore, prevents the model from predicting such kind of thermal crises: the simulated 
steady conditions and the hyper-gravity improvements, consequently, may differ from the experimental data.

\section{ACKNOWLEDGEMENTS}

The present work has been carried out in the framework of the project ESA-AO-2009 "Microgravity investigations of a novel two phase thermal management device for the ISS" financed by the Italian Space Agency (ASI-DOLFIN-II). The authors acknowledge the support of the European Space Agency through the MAP Condensation program (MAP ENCOM, AO-2004-096) as well as NOVESPACE team in Bordeaux and Dr. Vladimir Pletser from ESA for his support and encouragement in the parabolic flight campaigns. Thank to Dr. Olivier Minster and Dr. Balazs Toth for their interest and support to the PHP activities and to all the members of the Pulsating Heat Pipe International Scientific Team, led by Prof. M. Marengo, for their contribution in the pushing of the PHP technology for real space applications. In particular a thanks is due to Dr. Vadim Nikolayev for the very fruitful discussion regarding the phase changes modeling. Finally the authors acknowledge Dr. Miltos Petridis and the School of Computing, Engineering and Mathematics of the University of Brighton for the provided financial support.

\section{ANNEX A: MASS AND ENERGY BALANCES DURING PHASE CHANGES}

\section{A.1 Heterogeneous phase changes}

\section{Condensation.}

Figure $2 \mathrm{~A}$ shows in red the global control volume $C V$ adopted to solve mass and energy balances during heterogeneous condensation: it is closed, isochoric and diabatic with respect to the pipe walls. It contains three closed sub-systems, the $i$-th vapor plug $(V P)$ and two liquid slices $(L S)$, respectively the last and first of the $j$-th and of the $j+1$-th liquid slug. The closed single vapor subdomain allows a two-phase change and it is diabatic; the closed liquid sub-domains are mono-phase and adiabatic. The heterogeneous condensation will eventually occur in the vapor plug.

The mass and the energy balances written for the global control volume $C V$ are:

$\left\{\begin{array}{l}d m_{C V}=0 \\ d U_{C V}=q_{w f, V P} A_{w f, V P} d t \\ d V_{C V}=0\end{array}\right.$

where $V$ stands for volume.

Since it has been assumed that condensation is isothermal and isobaric (assumption $\mathrm{f}$ ), it cannot be isochoric at the same time. Thus, in order to satisfy Eq. 14, after condensation the vapor element will follow an isothermal expansion to occupy the volume freed by the part of vapor that has become liquid.

The mass and energy balance written for the vapor plug only, considering it a two-phase system are:

$\left\{\begin{array}{l}d m_{V P}=d\left(m_{l}+m_{v}\right)=0 \rightarrow d m_{l}=-d m_{v} \\ d U_{V P}=d\left(U_{l}+U_{v}\right)=\left(q_{w f, V P} A_{w f, V P}\right) d t-P d V_{V P}=Q d t-P d\left(V_{l}+V_{v}\right)\end{array}\right.$

where $Q=q_{w f, V P} A_{w f, V P}$.

The integration of the first row of Eq. 15 in time defined the mass $m_{L V, h e t}$ that will change phase in the time step; since vapor undergoes condensation $m_{L V \text {,het }}$ will be negative:

$\int_{m_{t_{i}}}^{m_{t_{i+1}}} d m_{v}=m_{v, t_{i+1}}-m_{v, t_{i}}=m_{L V, h e t}$ 
7

On the other hand, assuming that liquid is incompressible and accounting for the definition of enthalpy $(H=U+P V)$, the second row of Eq. 15 can be explicated as:

$m_{l} d u_{l}+m_{v} d u_{v}=Q d t-h_{l} d m_{l}-h_{v} d m_{v}+\frac{P V_{v}}{\rho_{v}} d \rho_{v}$

where $h_{l}$ and $h_{v}$ represent the specific enthalpy links to liquid and vapor respectively.

Introducing $d m_{l}=-d m_{v}$ in Eq. 17, it yields to:

$m_{l} d u_{l}+m_{v} d u_{v}=Q d t-h_{L V} d m_{v}+\frac{P V_{v}}{\rho_{v}} d \rho_{v}$

By integrating between the status in $t_{i}$ and in $t_{i+1}$, and assuming that the phase change is isothermal and isobaric (assumption f), Eq. 18 yields to Eq. 19 which exactly corresponds to Eq. 3:

$$
m_{L V, h e t}=\frac{Q}{h_{L V}} \Delta t=\frac{q_{w f, V P} A_{w f, V P}}{h_{L V}} \Delta t
$$

Since $m_{L V, \text { het }}$ is negative $q_{w f, V P}$ will exit the domain which is expected during condensation.

At this stage, the vapor sub-domain is containing both liquid and vapor. However, the numerical procedure adopted does not allow the existence of fluidic elements with such characteristic, since the starting thermodynamic state of the vapor plug needs to be always with quality equal to one. Thus the condensed mass will be merged with the adjacent liquid slices paying attention to conserve energy and mass in $C V$. In addition, in order to be consistent with the Eq. 14, the remaining vapor will undergo an isothermal expansion $\left(P V_{v}=\right.$ cost $)$.

By solving the mass and the energy balances during the merging operation:

$d m_{C V}=0 \rightarrow\left\{\begin{array}{l}m_{V P, t_{i+1}}=m_{V P, t_{i}}-\left|m_{L V, \text { het }, 1}\right|-\left|m_{L V, \text { het }, 2}\right| \\ m_{L S 1, t_{i+1}}=m_{L S 1, t_{i}}+\left|m_{L V, \text { het }, 1}\right| \\ m_{L S 2, t_{i+1}}=m_{L S 2, t_{i}}+\left|m_{L V, \text { het }, 2}\right|\end{array}\right.$

$d U_{C V}=0 \rightarrow\left\{\begin{array}{l}T_{V P, t_{i+1}}=T_{V P, t_{i}} \\ m_{L S 1, t_{i+1}} C_{V, l} T_{L S 1, t_{i+1}}=m_{L S 1, t_{i}} C_{V, l} T_{L S 1, t_{i}}+\left|m_{L V, h e t, 1}\right| C_{V, l} T_{V P, t_{i}} \\ m_{L S 2, t_{i+1}} C_{V, l} T_{L S 2, t_{i+1}}=m_{L S 2, t_{i}} C_{V, l} T_{L S 2, t_{i}}+\left|m_{L V, h e t, 2}\right| C_{V, l} T_{V P, t_{i}}\end{array}\right.$

$m_{L V, \text { het }, 1}$ is the mass that will merge with $m_{L S, 1}$, while $m_{L V, h e t, 2}$ is the mass that will merge with $m_{L S, 2}$.

Finally, imposing an isothermal expansion on the remaining vapor (considered ideal) Eq. 14 is completely satisfied:

$P_{V P, t_{i+1}} V_{V P, t_{i+1}}=m_{V P, t_{i+1}} R^{*} T_{V P, t_{i+1}}$

where $R^{*}$ is the gas constant. $V_{V P, t_{i+1}}$ should be chosen to satisfy $d V_{C V}=0$ and thus:

$V_{V P, t_{i+1}}=V_{V P, t_{i}}-\frac{m_{L S 1, t_{i+1}}}{\rho_{l}}-\frac{m_{L S 2, t_{i+1}}}{\rho_{l}}$

After this procedure for all the fluidic elements thermodynamic properties are updated to withstand the new thermodynamic status of the liquid and vapor elements at $t^{\prime}=t+\Delta t$.

\section{Evaporation.}

The solution adopted to solve heterogeneous evaporation follows the same modeling strategy used for condensation but it is applied to the control volumes of Fig 2B described in section 2.1.1. 


\section{Creation or disappearance of fluidic elements.}

Heterogeneous evaporation and condensation processes are solved consecutively moving along the fluidic path. The procedure described above showed mass exchange with the adjacent elements. However, it can occur that a new element is generated if $m_{L V \text {,het }}$ is higher than a minimum value $m_{\min }$ computed as the mass of an equivalent sphere which fits perfectly within the tube $\left(m_{\text {min,eva }(\text { cond })}=\frac{1}{6} \pi d_{i n}^{3} \rho_{v(l)}\right)$; on the other hand, if $m_{L V, \text { het }}$ exceeds the mass of the corresponding element, this one vanishes and the adjacent slugs/plugs merge. The above procedure can be followed even in these cases, but to guarantee the length conservation, the control volume $C V$ should be chosen adequately (Fig. 18) to assure that its global dimension does not change during the entire process.

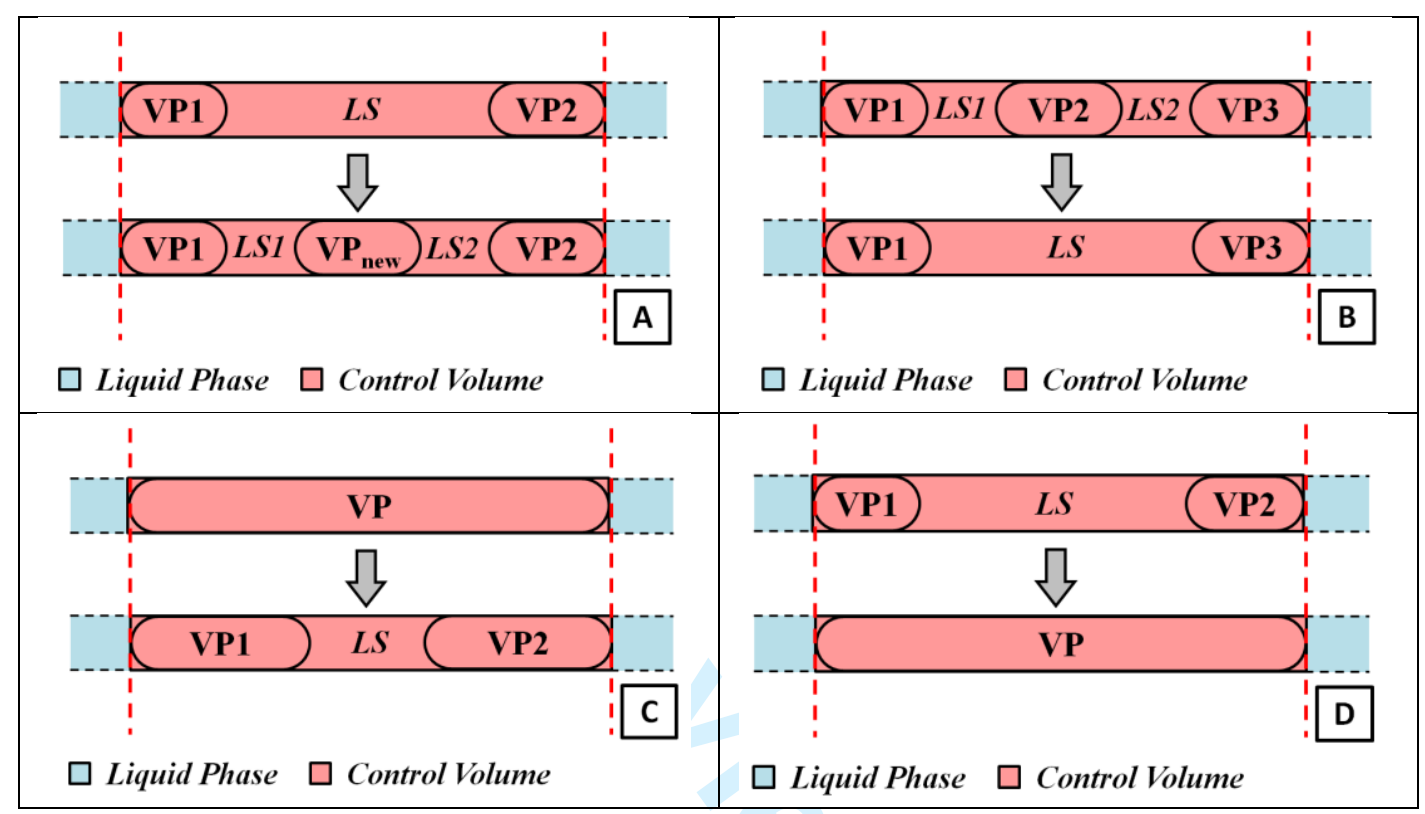

Fig. 18: Schematic of the fluidic control volumes $C V$ (in red) adopted in case of heterogeneous evaporation with generation of new a vapor plug (A), heterogeneous condensation with vapor plug disappearance (B), heterogeneous condensation with generation of new a liquid slug (C) and heterogeneous evaporation with liquid slug disappearance (D).

\section{A.2 Homogeneous phase changes through the interface}

For both evaporation and condensation, the adopted control volume $C V$ is shown in Fig. $2 \mathrm{~A}$. $C V$ is considered closed, adiabatic and isochoric. The two liquid slices $(L S)$ and the vapor plug $(V P)$ are open and adiabatic. However, if the control volume is adiabatic, it cannot be assumed that the phase changes are both isothermal and isobaric, thus only the assumption $d P=0$ has been maintained (assumption f). As done before for the heterogeneous phase changes, in order to assure that $d V_{C V}=0$, the homogeneous phase changes at the interface must be followed by isothermal expansions or compressions of the vapor sub-domain.

Condensation.

The mass and the energy balances written on the global control volume $C V$ are:

$\left\{\begin{array}{l}d m_{C V}=0 \\ d U_{C V}=0 \\ d V_{C V}=0\end{array}\right.$

Condensation will take place in the vapor phase, thus $V P$ is considered two-phase. The mass and energy balances written for this sub-domain are: 
$\left\{\begin{array}{l}d m_{V P}=d\left(m_{l}+m_{v}\right)=-\dot{m}_{\text {out }, l} d t \rightarrow d m_{l}=-d m_{v}-\dot{m}_{\text {out }, l} d t \\ d U_{V P}=d\left(U_{l}+U_{v}\right)=-P d V_{V P}-h_{l} \dot{m}_{\text {out }, l} d t=-P d\left(V_{l}+V_{v}\right)-h_{l} \dot{m}_{\text {out }, l} d t\end{array}\right.$

582 where $m_{\text {out }, l}$ is the condensed mass outgoing $V P$.

583 Assuming incompressible liquid and accounting for the definition of enthalpy $(H=U+P V)$, Eq.

858425 yields to:

$m_{l} d u_{l}+m_{v} d u_{v}=-h_{l} d m_{l}-h_{v} d m_{v}+\frac{P V_{v}}{\rho_{v}} d \rho_{v}-h_{l} \dot{m}_{o u t, l} d t$

Introducing $d m_{l}=-d m_{v}-\dot{m}_{o u t, l} d t$ in Eq. 26 and accounting for isobaric condensation:

$m_{l} d u_{l}+m_{v} d u_{v}+m_{v} R^{*} d T_{v}=-h_{L V} d m_{v}$

By integrating between the status in $t_{i}$ and $t_{i+1}$, Eq. 27 yields to Eq. 28:

$\left.m_{v, t_{i}} \Delta u_{v}\right|_{t_{i}} ^{t_{i}+1}+\left.R^{*} m_{v, t_{i}} \Delta T_{v}\right|_{t_{i}} ^{t_{i}+1}=-\left.h_{L V} \Delta m_{v}\right|_{t_{i}} ^{t_{i}+1}$

Since $-\left.h_{L V} \Delta m_{v}\right|_{t_{i}} ^{t_{i}+1}>0$, the vapor that is condensing undergoes a heating process. $\int_{u_{i}}^{u_{i}+1} m_{l} d u_{l}=$ $\left.m_{l, t_{i}} \Delta u_{l}\right|_{t_{i}} ^{t_{i}+1}=0$ since $V P$ does not contain any liquid at $t_{i}$. If the quality of the vapor plug at the end of the process is one, $\left.\Delta m_{v}\right|_{t_{i}} ^{t_{i}+1}$ represent the mass of the condensed vapor $m_{L V, h o m}$, which is still unknown: $\left.m_{v, t_{i}} \Delta u_{l}\right|_{t_{i}} ^{t_{i}+1}+\left.R^{*} m_{v, t_{i}} \Delta T_{v}\right|_{t_{i}} ^{t_{i}+1}=h_{L V}\left|m_{L V, h o m}\right|$.

The condensed mass, then, flows inside the two $L S s$. This allows $d m_{C V}=0$ since $\left.\sum_{\xi=1}^{2} \Delta m_{L S \xi}\right|_{t_{i}} ^{t_{i}+1}=\sum_{\xi=1}^{2}\left|m_{L V, h o m, \xi}\right|=\left|m_{L V, h o m}\right|$. In order to conserve the internal energy on each $L S$, and thus on $C V$, one should account for:

$d U_{L S \xi}=d\left(H_{L S \xi}-P V_{l \xi}\right)=-P d V_{l \xi}+h_{l \xi} d m_{i n \xi} \quad \xi=1 \div 2$

Assuming the process isobaric and integrating between the initial and final status, for each LS:

$\int_{H_{t_{i}}}^{H_{t_{i+1}}} d H_{L S \xi}=\left.h_{l \xi}\left|m_{L V, h o m, \xi}\right| \rightarrow \Delta\left(m_{l} h_{l}\right)\right|_{t_{i}} ^{t_{i}+1}=h_{l \xi}\left|m_{L V, h o m, \xi}\right|$

In addition, in order to make Eq. 24 true, the vapor element will undergo an isothermal expansion:

$P_{V P, t_{i+1}} V_{V P, t_{i+1}}=m_{V P, t_{i+1}} R^{*} T_{V P, t_{i+1}}$

Differently from heterogeneous phase changes, this time the unknown is $m_{L V, h o m}$ since $P_{V P, t_{i+1}}=$ $P_{s a t} ; V_{V P, t_{i+1}}$ is chosen, on the other hand, to satisfy $d V_{C V}=0$ (Fig. 3A):

$V_{V P, t_{i+1}}=V_{V P, t_{i}}-\frac{\left|m_{L V, h o m}\right|}{\rho_{l}}$

After this procedure for all the fluidic elements thermodynamic properties are updated to withstand the new thermodynamic status of the liquid and vapor elements at $t^{\prime}=t+\Delta t$.

\section{Evaporation.}

The solution adopted to solve homogeneous evaporation through the interface follows the same modeling strategy used for condensation. However, in this case evaporation will take place in the liquid phase, thus Eq. 25 should be explicated for each $L S$, while Eq. 29 for the vapor plug. 


\section{Latin symbols}

\begin{tabular}{|c|c|c|c|}
\hline$A$ & Cross flow area, $\left[\mathrm{m}^{2}\right]$ & $U$ & Internal energy, $[\mathrm{J}]$ \\
\hline$A_{\text {ex }}$ & External lateral area, $\left[\mathrm{m}^{2}\right]$ & $u$ & Specific internal energy, $\left[\mathrm{Jkg}^{-1}\right]$ \\
\hline$A_{w f}$ & Internal lateral area, $\left[\mathrm{m}^{2}\right]$ & $V$ & Volume, $\left[\mathrm{m}^{3}\right]$ \\
\hline$a$ & Acceleration, $\left[\mathrm{ms}^{-2}\right]$ & $w$ & Velocity, $\left[\mathrm{ms}^{-1}\right]$ \\
\hline$c_{V}$ & Specific heat constant volume, $\left[\mathrm{Jkg}^{-1} \mathrm{~K}^{-1}\right]$ & $x$ & Axial coordinates, $[\mathrm{m}]$ \\
\hline$d_{\text {in }}$ & Diameter, $[\mathrm{m}]$ & $y$ & Axial coordinates, $[\mathrm{m}]$ \\
\hline$f_{\tau}$ & Friction coefficient, [] & \multicolumn{2}{|c|}{ Greek symbols } \\
\hline$g$ & Gravity acceleration, $\left[\mathrm{ms}^{-2}\right]$ & $\Delta t$ & Time step, [s] \\
\hline$H$ & Enthalpy, $[\mathrm{J}]$ & $\vartheta$ & Inclination to horizontal, [rad] \\
\hline$h$ & Convection coefficient, $\left[\mathrm{Wm}^{-2} \mathrm{~K}^{-1}\right]$ & $\rho$ & Density, $\left[\mathrm{kg} / \mathrm{m}^{3}\right]$ \\
\hline$h_{l}$ & Liquid specific enthalpy, $\left[\mathrm{Jkg}^{-1}\right]$ & \multicolumn{2}{|c|}{ Subscripts } \\
\hline$h_{v}$ & Vapor specific enthalpy, $\left[\mathrm{Jkg}^{-1}\right]$ & $f$ & Fluidic \\
\hline$h_{L V}$ & Latent heat of vaporization, $\left[\mathrm{Jkg}^{-1}\right]$ & hom & Homogenous phase change \\
\hline$k$ & Thermal conductivity, $\left[\mathrm{Wm}^{-1} \mathrm{~K}^{-1}\right]$ & het & Heterogeneous phase change \\
\hline$L$ & Length, $[\mathrm{m}]$ & $l$ & Liquid \\
\hline$m$ & Mass, $[\mathrm{kg}]$ & sat & Saturated conditions \\
\hline$m_{L V}$ & Evaporated/Condensed mass, $[\mathrm{kg}]$ & $v$ & Vapor \\
\hline$N$ & Number of elements, [] & $w$ & Wall \\
\hline$p$ & Pressure, $[\mathrm{Pa}]$ & $\infty$ & Environmental \\
\hline$Q$ & Heat power, [W] & \multicolumn{2}{|c|}{ Acronyms } \\
\hline$q_{e x}$ & External heat flux, $\left[\mathrm{Wm}^{-2}\right]$ & $\mathrm{CV}$ & Control Volume \\
\hline$q_{w f}$ & Heat flux between wall and fluid, $\left[\mathrm{Wm}^{-2}\right]$ & ID & Internal Diameter \\
\hline$R^{*}$ & Gas constant, $\left[\mathrm{Jkg}^{-1} \mathrm{~K}^{-1}\right]$ & $\mathrm{LS}$ & Liquid Slug \\
\hline$R a$ & Rayleigh number, [] & ESA & European Space Agency \\
\hline$R e$ & Reynolds number, [] & OD & Outer Diameter \\
\hline$T$ & Temperature, $[\mathrm{K}]$ & PHP & Pulsating Heat Pipe \\
\hline$t$ & Time, $[\mathrm{s}]$ & VP & Vapor Plug \\
\hline
\end{tabular}

\section{REFERENCES}

[1] DeLombard R, Hrovat K, Kelly E, McPherson K. Microgravity environment on the international space station. Proc. of the 42nd Aerospace Sciences Meeting and Exhibit 2004.

[2] Khandekar S, Groll M. Roadmap to realistic modeling of closed loop pulsating heat pipes. Proc. of the 9th Int. Heat Pipe Symposium 2008.

[3] Reay DA, McGlen RJ, Kew PA. Heat pipes: theory, design and applications. Elsevier Ltd, 2006. ISBN: 978-O-08-098266-3.

[4] Akachi H. US Patent No. 4921041. 1990.

[5] Akachi, H. US Patent No. 5219020. 1993.

[6] Mameli M, Marengo M, Khandekar S. Local heat transfer measurement and thermo-fluid characterization of a pulsating heat pipe. Int. J. of Thermal Sciences 2014; 45: 140-152.

[7] Kiseev VM, Zolkin KA. The influence of acceleration on the performance of oscillating heat pipe. Proc. of the 11th International Heat Pipe Conference 1999.

[8] Van Es J, Woering AA. High-acceleration performance of the flat swinging heat pipe. NLR-TP2000-265 2000

[9] Gu J, Kawaji M, Futamaca R. Effect of gravity on the performance on pulsating heating pipe. $J$. of Thermophysics and Heat Transfer 2004; 18: 370-378. 
1624

[10] Gu J, Kawaji M, Futamata R. Microgravity performance of micro pulsating heat pipe, Microgravity Sci. Technol 2005; 16: 181-185.

[11] Mameli M, Manzoni M, Araneo L, Filippeschi S, Marengo M. Pulsating heat pipe in hypergravity conditions. Heat Pipe Science and Techonology, an International Journal. 2014 ACCEPTED FOR PUBLICATION.

[12] Mameli M, Araneo L, Filippeschi S, Marelli L, Testa R, Marengo M. Thermal response of a closed loop pulsating heat pipe under variable gravity field, Int. J. of Thermal Sciences 2014; 80: $11-22$.

[13] Mameli M, Marelli L, Manzoni M, Araneo L, Filippeschi A, Marengo M. Closed loop pulsating heat pipe: ground and microgravity experiments, Proc. Of the 9th International Conference on two-phase systems for ground and space applications 2014

[14] Mangini D, Mameli M, Georgoulas A, Araneo L, Filippeschi S, Marengo M. A pulsating heat pipe for space applications: ground and microgravity experiments. Int. Journal of Thermal Science 2015; 95: 55-63.

[15]Zhang Y, Faghri A. Advances and unsulved issues in pulsating heat pipes. Heat Transfer Engineering 2008; 29: 20-44.

[16] Tang X, Sha L, Zhang H, Ju Y. A review of recent experimental investigations and theoretical analyses for pulsating heat pipes. Front. Energy 2013; 7: 161-173.

[17] Miyazaki Y, Akachi H, Polasek F, Stulk P. Heat transfer characteristics of looped capillary heat pipe. Proc. of the 5th Int. Heat Pipe Symposium 1996.

[18] Miyazaki Y, Akachi H. Self excited oscillation of slug flow in a micro channel. Proc. of the 3rd Int. Conf. on Multiphase Flow 1998.

[19]Zuo ZJ, North MT, Ray L. Combined pulsating and capillary heat pipe mechanism for cooling of high heat flux electronics, Proc. ASME Heat Transfer Device Conference 1999.

[20] Zuo ZJ, North MT, Wert KL. High heat flux heat pipes for cooling of electronics. IEEE Transactions on Components and Packaging Technologies 2001; 24-2: 220-225.

[21] Spinato G, Borhani N, Thome JR, Understanding the self-sustained oscillating two-phase flow motion in a closed loop pulsating heat pipe, Energy 2015; 90: 889-899.

[22] Khandekar S, Cui X, Groll M. Thermal performance modeling of pulsating heat pipe by artificial neural networks, Proc. of 12th Int. Heat Pipe Conf 2002.

[23] Chen PH, Lee YW, Chang TL Predicting thermal instability in a closed loop pulsating heat pipe system, Appl. Therm. Eng. 2009; 29-8: 1566-1576.

[24] Lee YW, Chang TL. Application of NARX neural networks in thermal dynamics identification. Energy Convers. Manage 2009; 50-4: 1069-1078.

[25] Liu XD, Hao YL. Numerical simulation of vapor-liquid two-phase flow in a closed loop oscillating heat pipe. Proc. of the ASME 2009 International Mechanical Engineering Congress and Exposition 2009.

[26] Lin Z, Wang S, Shirakashi R, Zhang LW. Simulation of a miniature oscillating heat pipe in bottom heating mode using CFD with unsteady modeling. Int. Journal of Heat and Mass Transfer 2013; 57: 642-656.

[27] Holley B, Faghri A. Analysis of pulsating heat pipes with capillary wick and varying channel diameter. Int. J. Heat Mass Transfer 2005; 48: 2635-2651.

[28] Mameli M, Marengo M, Zinna S. Thermal simulation of a pulsating heat pipe: effects of different liquid properties on a simple geometry. Heat Transfer Engineering 2012; 33: 1177 1187.

[29] Mameli M, Marengo M, Zinna S. Numerical model of a multi-turn closed loop pulsating heat pipe: effect of the local pressure losses due to meanderings. Int. J. Heat Mass Transfer 2012; 55: 1036-1047.

[30] Mameli M, Marengo M, Zinna S. Numerical investigation of the effects of orientation and gravity in a closed loop pulsating heat pipe. Microgravity Science and Technology 2012; 24-2: 79-92.

[31] Hairer E, Wanner G. Solving Ordinary Differential Equations II. Stiff and DifferentialAlgebraic Problems. Springer, 1996. 
677 678 679 5680 6681 7682 8683 96884 11685 12686 13687 14688 15689 17690 18691 19 20 21 22 23 24 25 26 27 28 29 30 31 32 33 34 35 36 37 38 39 40 41 42 43 44 45 46 47 48 49 50 51 52 53 54 55 56 57 58 59 60

[32] Hairer E, Lubich C, Wanner G. Geometic numerical integration illustrated by the StörmerVerlet method. Acta Numerica 2003; 12: 399-450.

[33] Incropera F P, DeWitt D P. Fundamentals of Heat and Mass Transfer. Wiley, 2007.

[34] Haaland S. Simple and explicit formulas for the friction factor in turbulent flow. Transactions of ASME, Journal of Fluids Engineering 1983; 103: 89-90.

[35] Darby R. Correlate pressure drop through fittings. Chemical Engineering 2001; 104:127-130.

[36] Shah RK, London AL. Laminar flow forced convection in ducts. Advanced in Heat Transfer 1978.

[37] Das S, Nikolayev V, Lefèvre F, Pottier B, Khandekar S, Bonjour J. Thermally induced twophase oscillating flow inside a capillary tube. Int. J. Heat Mass Transfer 2010; 53: 3905-3913.

[38] Nikolayev VS. A dynamic film model of the pulsating heat pipe, J. Heat Transfer 2011; 133-8.

[39] Han Y, Shikazono N. Measurement of the liquid film thickness in micro tube slug flow. International Journal of Heat and Fluid Flow 2009; 30: 82-853.

[40] Shafii MB, Faghri A, Zhang Y. Thermal modeling of unlooped and looped pulasating heat pipes. Journal of Heat Transfer 2001; 123: 1159-1172. 


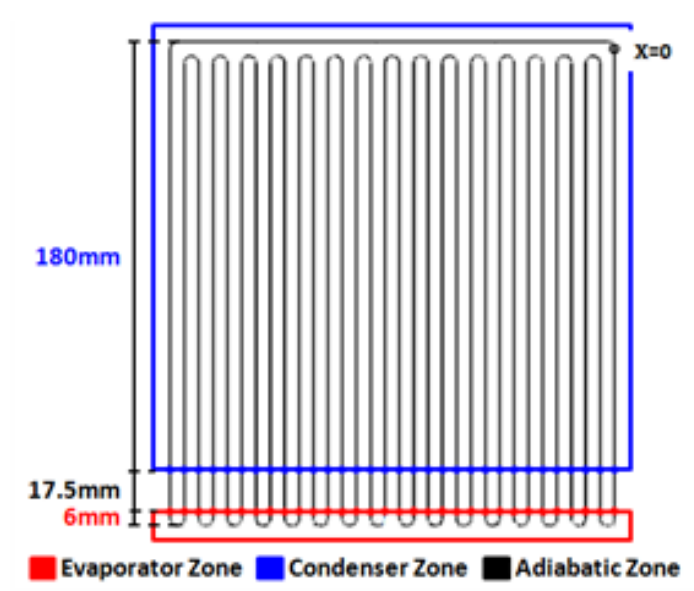

\begin{tabular}{lr}
\hline Input parameter & Value \\
\hline Working fluid & FC-72 \\
Volumetric filling ratio & 0.50 \\
Tube material & Copper \\
Internal diameter & $1.1 \mathrm{~mm}$ \\
External diameter & $2.0 \mathrm{~mm}$ \\
Surface roughness & $50 \mu \mathrm{m}$ \\
Total length & $6.62 \mathrm{~m}$ \\
$\mathrm{~N}^{\circ}$ of evaporator bends & 16 \\
$*$ External heat transfer coefficient & $400 \mathrm{~W} / \mathrm{m}^{2} \mathrm{~K}$ \\
$*$ Film Thickness & $50 \mu \mathrm{m}$ \\
$* \Delta T$ superheat & $2.5^{\circ} \mathrm{C}$ \\
$* \Delta T$ cooling & $0.01^{\circ} \mathrm{C}$ \\
$* \mathrm{~N}^{\circ}$ of slugs/plugs at $t=0$ & 20 \\
$* \mathrm{~N}^{\circ}$ of wall grids & 400 \\
\hline itivity analyses shown independency of the model \\
\end{tabular}

Tab. 1: Features of the tested PHP. Geometrical, physical and numerical input parameters are given. 
Fig. 1: Schematic of the numerical domains. The liquid slugs sub-domains are not explicitly indicated. The wall is considered completely wettable. $8 \times 1 \mathrm{~mm}(600 \times 600 \mathrm{DPI})$ 


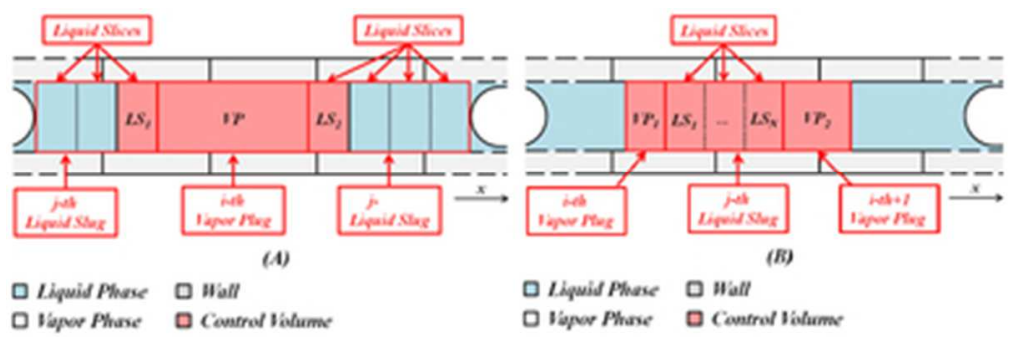

Fig. 2: Schematic of the fluidic control volumes CV (in red) adopted in case of heterogeneous condensation (A) or evaporation (B).

$16 \times 5 \mathrm{~mm}(600 \times 600 \mathrm{DPI})$ 
Fig. 3: Schematic of homogeneous condensation (A) and evaporation (B). Since the cross sectional area is constant, $\mathrm{V}=\mathrm{AL}$. $14 \times 5 \mathrm{~mm}(600 \times 600 \mathrm{DPI})$ 
1

2

3

4

5

6

7

8

9

10

11

12

13

14

15

16

17

18

19

20

21

22

23

24

25

26

27

28

29

30

31

32

33

34

35

36

37

38

39

40

41

42

43

44

45

46

47

48

49

50

51

52

53

54

55

56

57

58

59

60

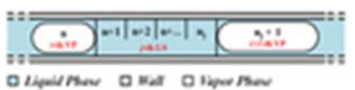

Fig. 4: Schematic of the fluidic domains and sub-domains accounted for the energy balance computation. VP stands for vapor plug, LS stands for liquid slug. $5 \times 1 \mathrm{~mm}(600 \times 600 \mathrm{DPI})$ 


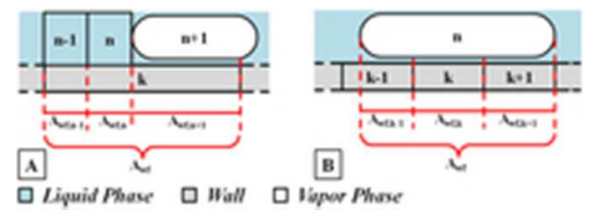

Fig. 5: Definition of $A_{w f}, A_{w f, n}$ and $A_{w f, k}$ from the wall point of view $(A)$ and from the fluid point of view (B). $9 \times 3 \mathrm{~mm}(600 \times 600 \mathrm{DPI})$ 
Fig. 6: Experimental and numerical evolution of the local spatial average temperatures for a bottom heated mode PHP at 50W. Solid lines represent mean temperatures; colored regions indicate the standard deviation on the recorder data. $80 \times 29 \mathrm{~mm}(600 \times 600 \mathrm{DPI})$ 

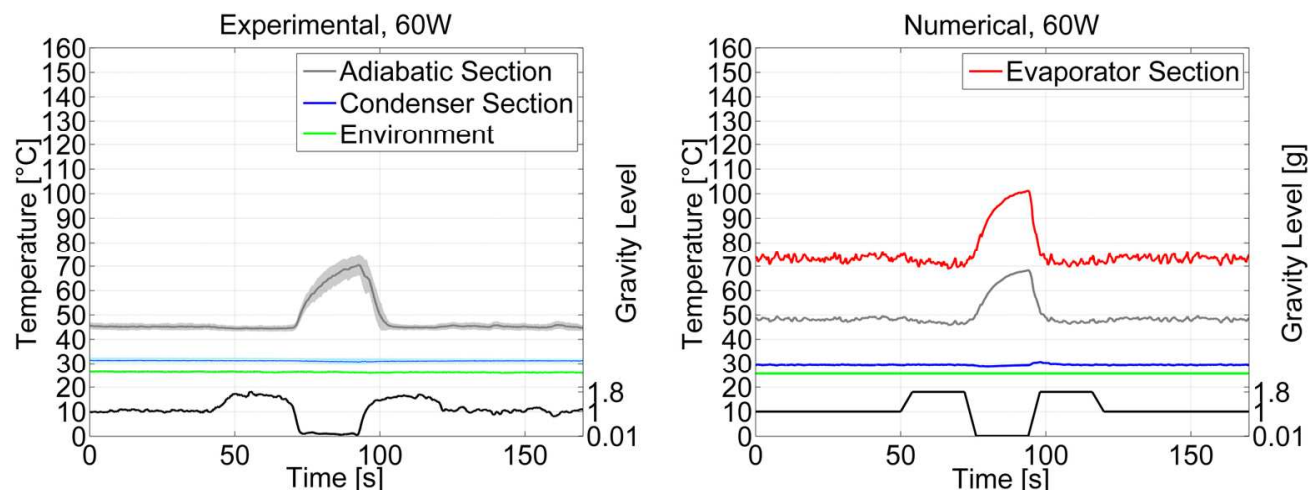

Fig. 7: Experimental and numerical evolution of the local spatial average temperatures for a bottom heated mode PHP at 60W. Solid lines represent mean temperatures; colored regions indicate the standard deviation on the recorder data. $80 \times 29 \mathrm{~mm}(600 \times 600 \mathrm{DPI})$ 
Fig. 8: Experimental and numerical evolution of the local spatial average temperatures for a bottom heated mode PHP at 70W. Solid lines represent mean temperatures; colored regions indicate the standard deviation on the recorder data. $80 \times 29 \mathrm{~mm}(600 \times 600 \mathrm{DPI})$ 

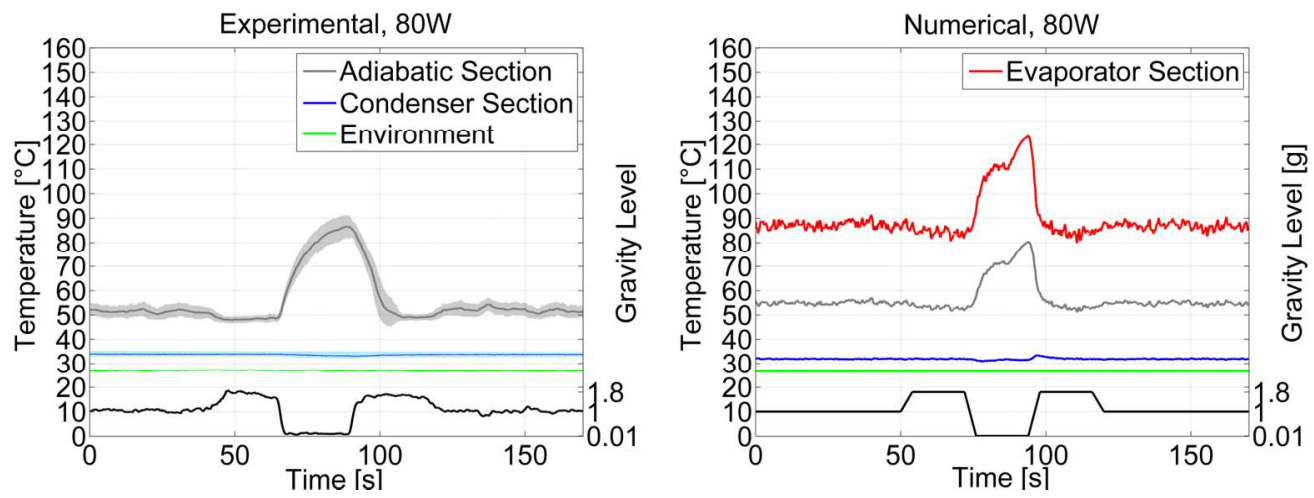

Fig. 9: Experimental and numerical evolution of the local spatial average temperatures for a bottom heated mode PHP at $80 \mathrm{~W}$. Solid lines represent mean temperatures; colored regions indicate the standard deviation on the recorder data. $80 \times 29 \mathrm{~mm}(600 \times 600 \mathrm{DPI})$ 
2

3

4

5

6

7

8

9

10

11

12

13

14

15

16

17

18

19

20

21

22

23

24

25

26

27

28

29

30

31

32

33

34

35

36

37

38

39

40

41

42

43

44

45

46

47

48

49

50

51

52

53

54

55

56

57

58

59

60
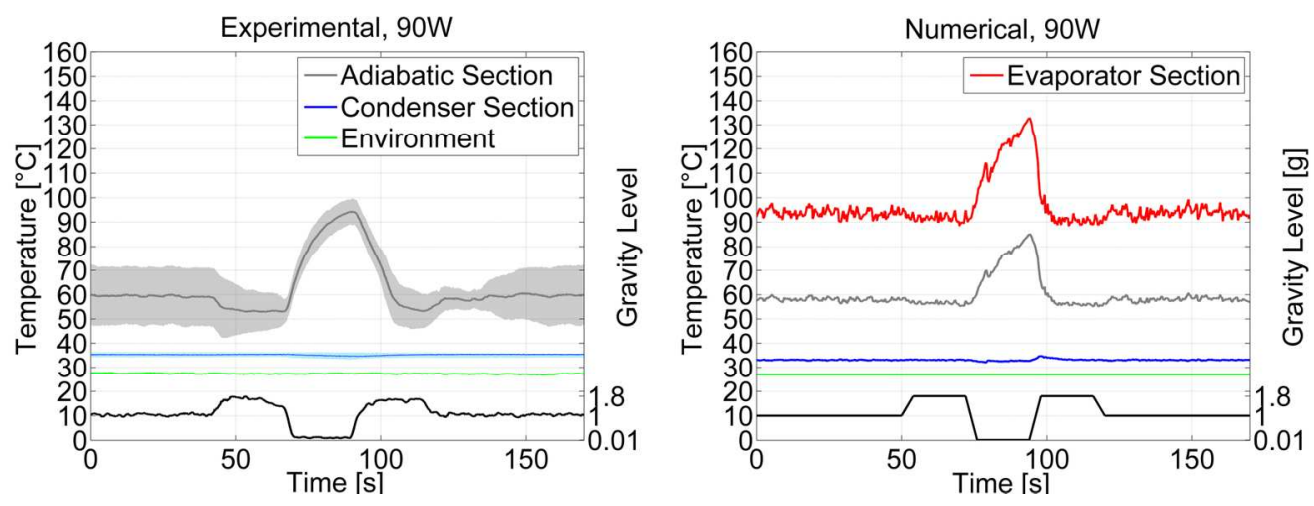

Fig. 10: Experimental and numerical evolution of the local spatial average temperatures for a bottom heated mode PHP at 90W. Solid lines represent mean temperatures; colored regions indicate the standard deviation on the recorder data. $80 \times 29 \mathrm{~mm}(600 \times 600 \mathrm{DPI})$ 

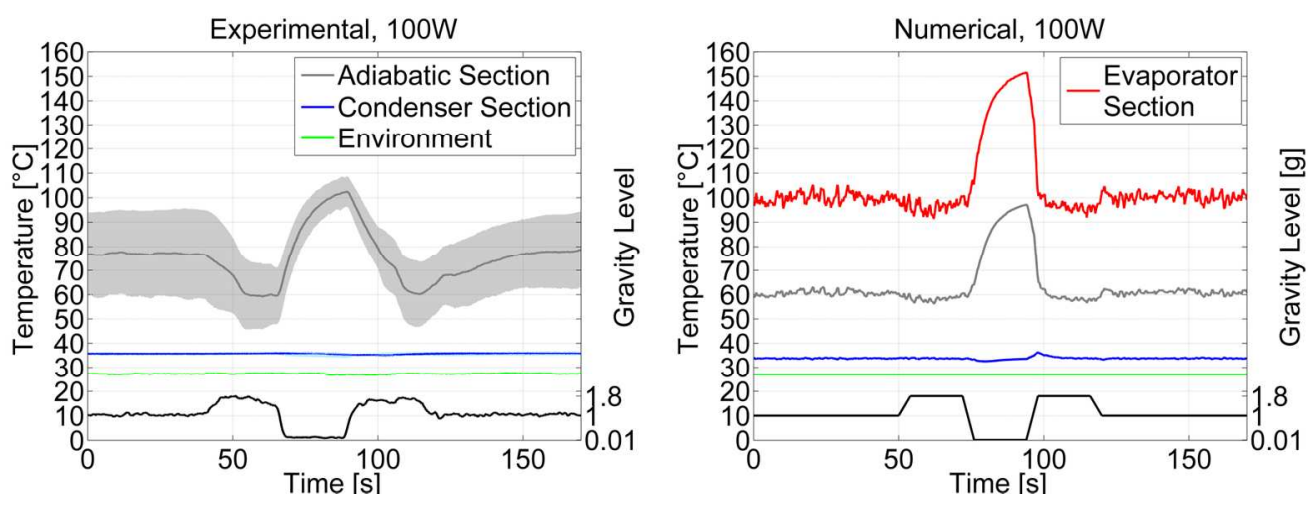

Fig. 11: Experimental and numerical evolution of the local spatial average temperatures for a bottom heated mode PHP at 100W. Solid lines represent mean temperatures; colored regions indicate the standard deviation on the recorder data. $80 \times 29 \mathrm{~mm}(600 \times 600 \mathrm{DPI})$ 


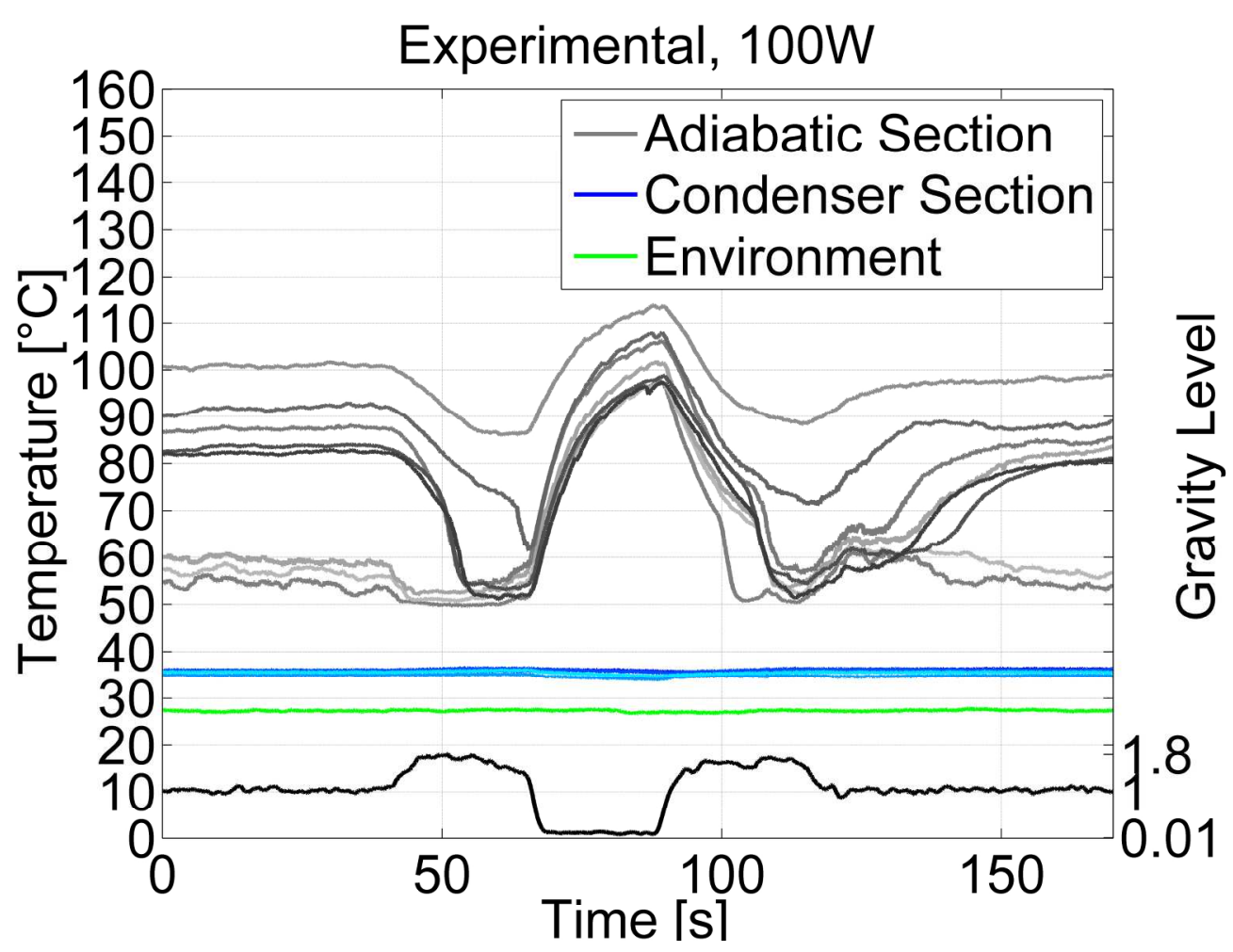

Fig. 12: Experimental wall temperatures for a bottom heated mode PHP at 100W. Greyish colours represent the adiabatic zone, bluish indicate the condenser section.

$$
101 \times 76 \mathrm{~mm}(600 \times 600 \mathrm{DPI})
$$



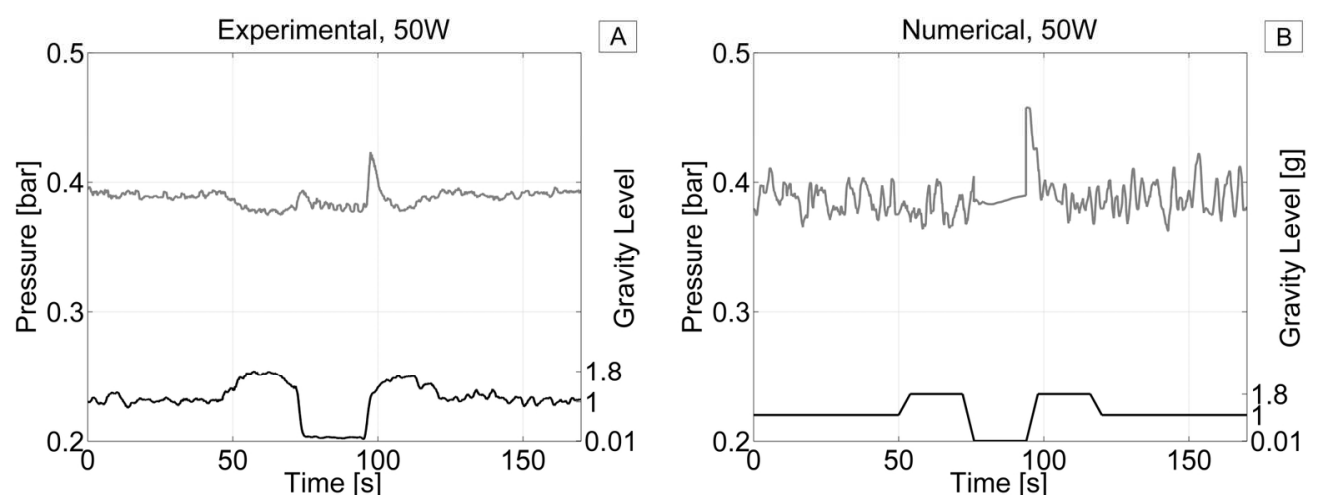

Fig. 13: Experimental (A) and numerical (B) pressure signal for the 50W parabola. $80 \times 29 \mathrm{~mm}(600 \times 600 \mathrm{DPI})$ 


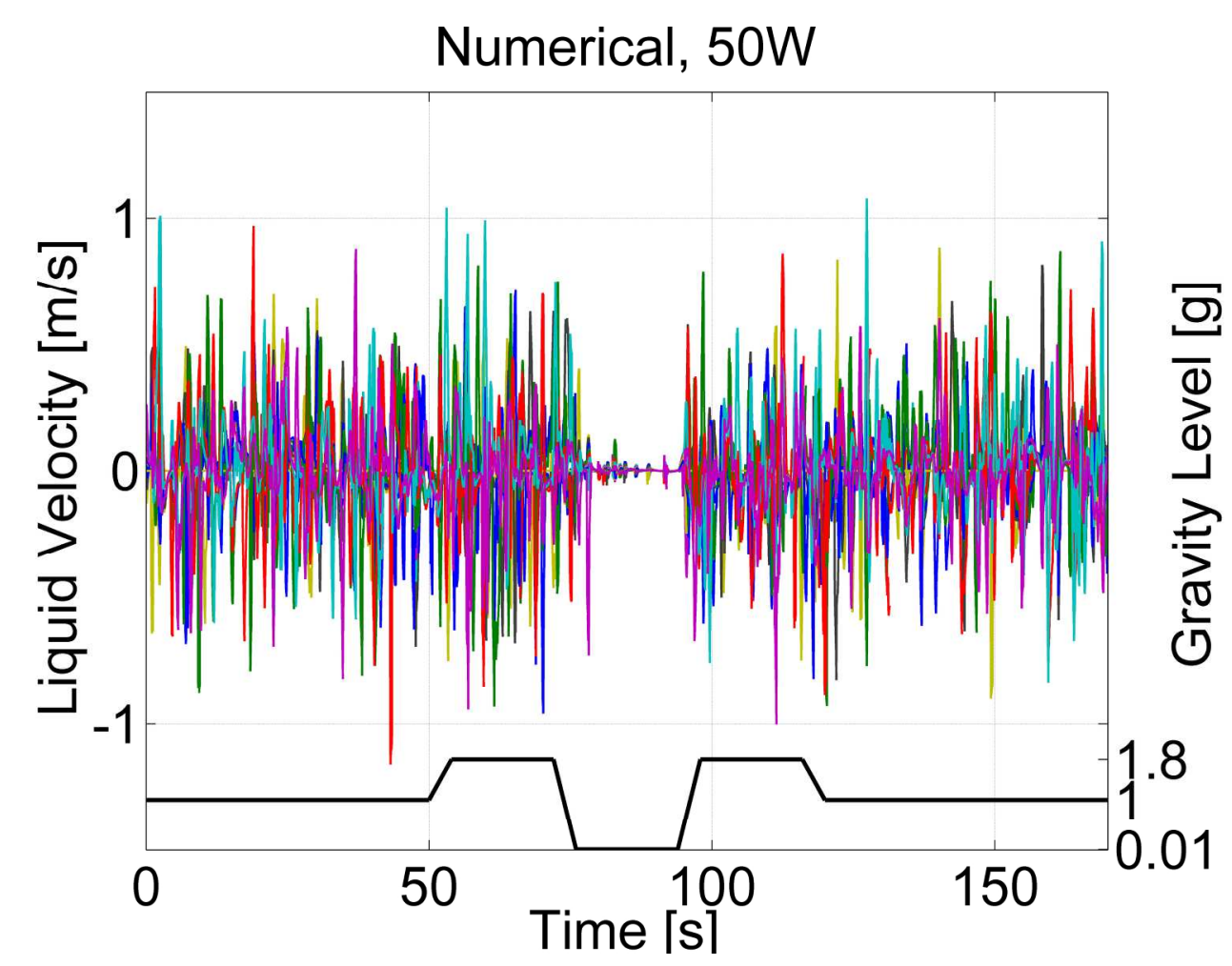

Fig. 14: Numerical computed liquid slugs velocities for the 50W parabola. Different colours indicate different elements.

$101 \times 76 \mathrm{~mm}(600 \times 600 \mathrm{DPI})$ 


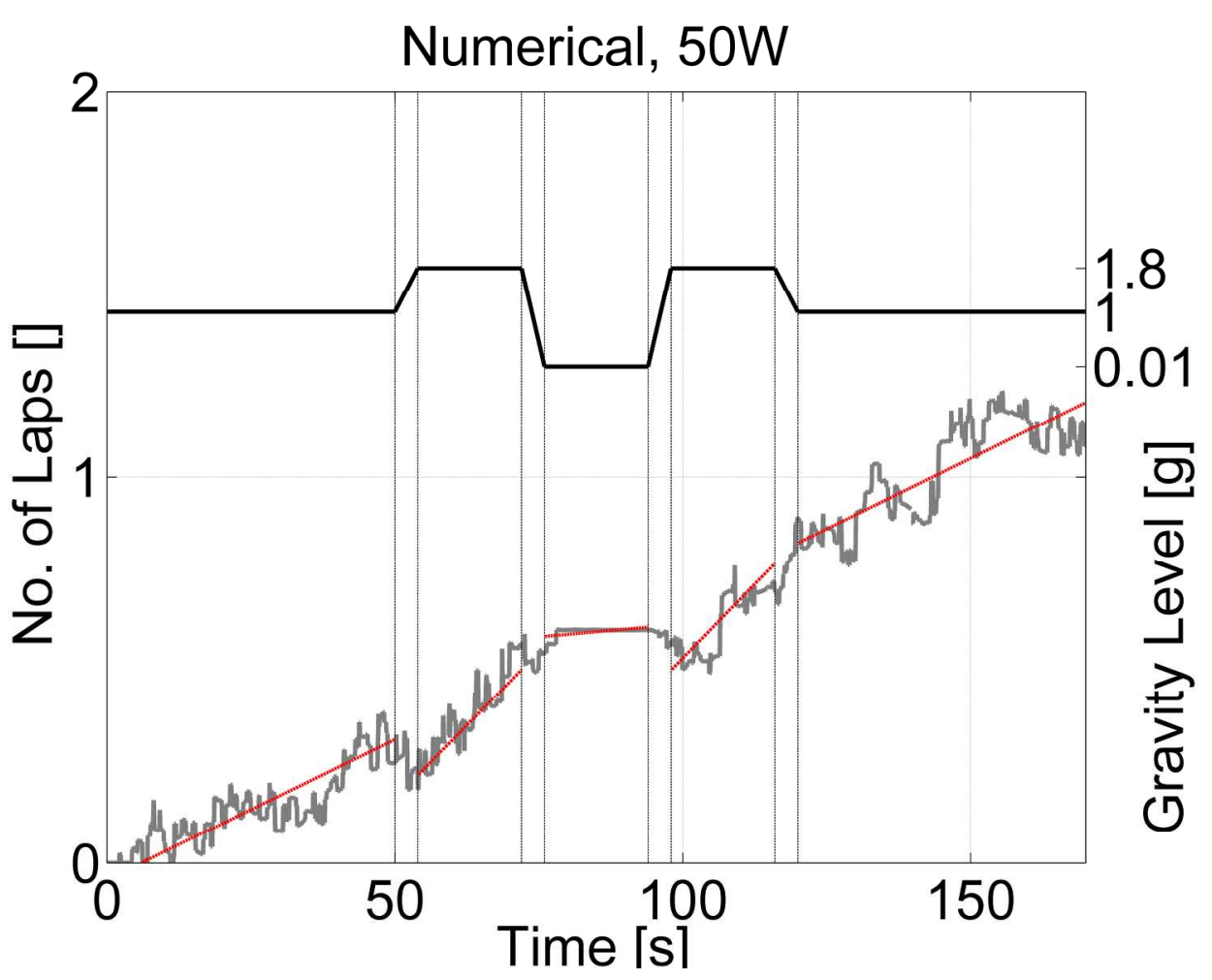

Fig. 15: 50W parabola; number of fluidic laps. Red dotted lines report the linear interpolation curves. $101 \times 76 \mathrm{~mm}(600 \times 600 \mathrm{DPI})$ 
Fig. 16: Numerical evolution of the local spatial average temperatures for the $50 \mathrm{~W}$ parabola (on the left) and the $50 \mathrm{~W}$ tilting manoeuvre (on the right). $80 \times 29 \mathrm{~mm}(600 \times 600$ DPI $)$ 

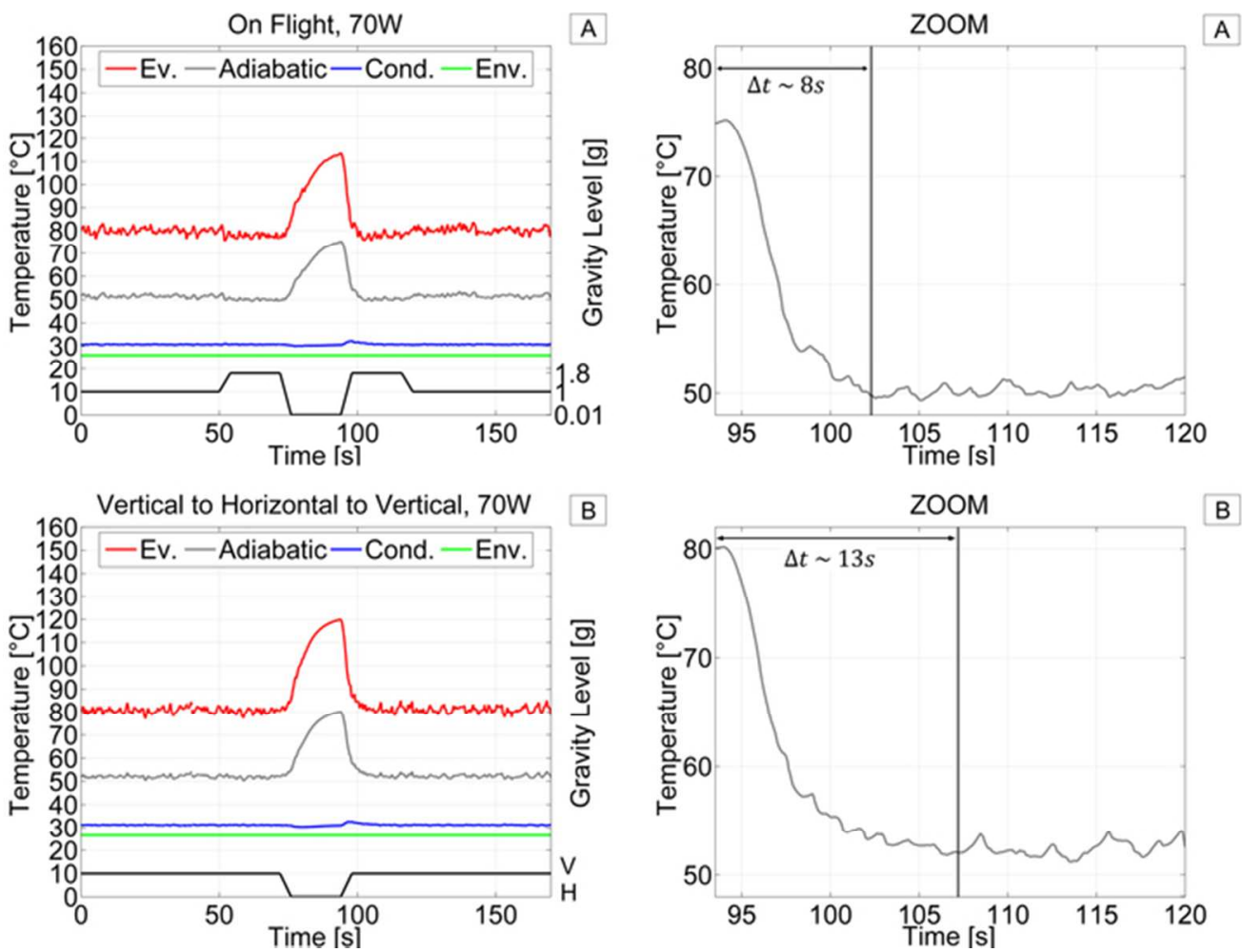

Fig. 17: Numerical evolution of the local spatial average temperatures for the $70 \mathrm{~W}$ parabola $(\mathrm{A})$ and the 70W tilting manoeuvre (B). On the right, zoom of the adiabatic temperature in the transition zone. $30 \times 22 \mathrm{~mm}(600 \times 600 \mathrm{DPI})$ 
Fig. 18: Schematic of the fluidic control volumes CV (in red) adopted in case of heterogeneous evaporation with generation of new a vapor plug $(A)$, heterogeneous condensation with vapor plug disappearance (B), heterogeneous condensation with generation of new a liquid slug (C) and heterogeneous evaporation with liquid slug disappearance (D). $23 \times 13 \mathrm{~mm}(600 \times 600 \mathrm{DPI})$ 


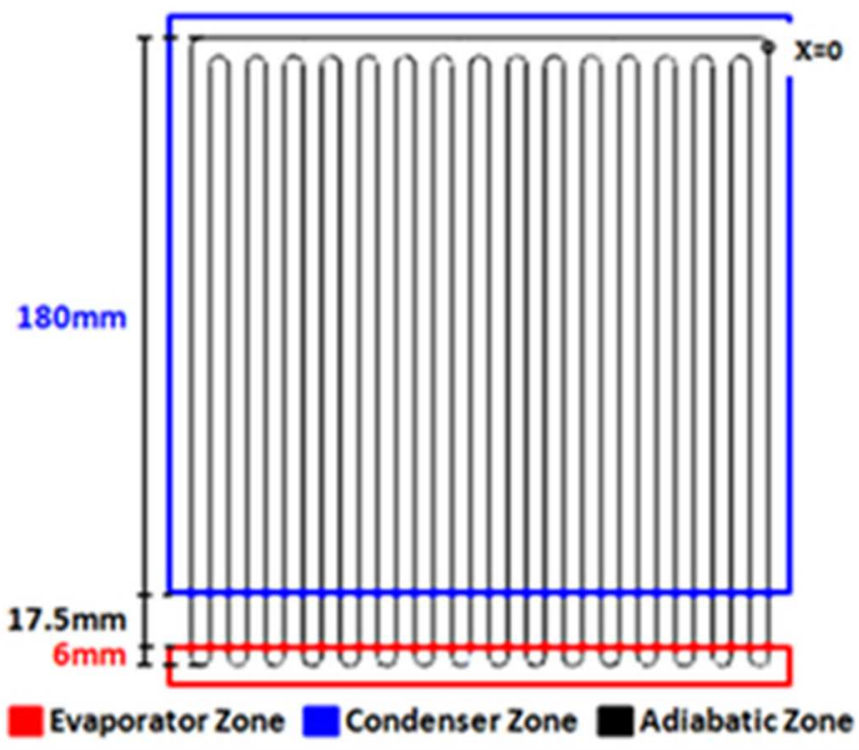

$14 \times 12 \mathrm{~mm}(600 \times 600 \mathrm{DPI})$

http://mc.manuscriptcentral.com/fluids 